\title{
The ER cholesterol sensor SCAP promotes CARTS biogenesis at ER-Golgi contact
} sites

Yuichi Wakana $^{1 *}$, Kaito Hayashi ${ }^{1}$, Takumi Nemoto ${ }^{1}$, Chiaki Watanabe ${ }^{1}$, Masato Taoka $^{2}$, Felix Campelo ${ }^{3}$, Hidetoshi Kumata ${ }^{1}$, Tomonari Umemura ${ }^{1}$, Hiroki Inoue ${ }^{1}$, Kohei Arasaki ${ }^{1}$, and Mitsuo Tagaya ${ }^{1 *}$

${ }^{1}$ School of Life Sciences, Tokyo University of Pharmacy and Life Sciences, Hachioji, Tokyo 192-0392, Japan.

${ }^{2}$ Faculty of Science, Department of Chemistry, Tokyo Metropolitan University, Hachioji, Tokyo 192-0397, Japan.

${ }^{3}$ ICFO-Institut de Ciencies Fotoniques, The Barcelona Institute of Science and Technology, Barcelona, Spain

Running title: A role of SCAP in CARTS biogenesis

*Corresponding authors:

Yuichi Wakana and Mitsuo Tagaya

E-mail: ywakana@toyaku.ac.jp, Phone: +81 42676 7116, Fax: +81 426768866

K. Hayashi and T. Nemoto contributed equally to this paper

The number of characters: 39,555

Summary: SCAP is the key regulatory protein in cholesterol metabolism. Wakana et al. describe a new role of SCAP in controlling Golgi PI4P turnover and the biogenesis of the Golgi-derived transport carries CARTS via cholesterol/PI4P exchange machinery at ER-Golgi contact sites. 


\section{Abstract}

In response to cholesterol deprivation, SCAP escorts SREBP transcription factors from the endoplasmic reticulum (ER) to the Golgi complex for their proteolytic activation, leading to gene expression for cholesterol synthesis and uptake. Here we show that in cholesterol-fed cells ER-localized SCAP interacts through Sac1 phosphoinositide 4-phosphate (PI4P) phosphatase with a VAP/OSBP complex, which mediates counter-transport of ER cholesterol and Golgi PI4P at ER-Golgi contact sites. SCAP knockdown inhibited the turnover of PI4P perhaps due to a cholesterol transport defect and altered the subcellular distribution of the VAP/OSBP complex. As in the case of perturbation of lipid transfer complexes at ER-Golgi contact sites, SCAP knockdown inhibited the biogenesis of the transGolgi network-derived transport carriers CARTS, which was reversed by expression of wild-type SCAP but not cholesterol sensing-defective mutants. Altogether, our findings reveal a new role of SCAP under cholesterol-fed conditions in the facilitation of CARTS biogenesis at ER-Golgi contact sites, depending on the ER cholesterol. 


\section{Introduction}

Cholesterol plays a crucial role in regulating the functions of mammalian cell membranes by determining their integrity and fluidity. Cholesterol together with sphingolipids can form liquid-ordered membrane nanodomains which are segregated from other lipids and thus are proposed to serve as platforms for specific proteins that regulate signal transduction and endocytosis at the plasma membrane (PM), and apical transport from the trans-Golgi network $(\mathrm{TGN})^{1-8}$. Increasing evidence -including recent experiments where sphingomyelin (SM) metabolism at the trans-Golgi membranes was perturbed-strongly suggests that such lipid nanodomains are required for the functional organization of enzymatic domains, cargo sorting, and transport carrier biogenesis at the $\mathrm{TGN}^{9-14}$, and thereby are crucial to maintain homeostatic control of the Golgi function.

De novo biosynthesis of cholesterol occurs at the endoplasmic reticulum (ER), where the key regulatory protein in cholesterol metabolism, called sterol regulatory element binding protein (SREBP) cleavage-activating protein (SCAP), localizes. SCAP is a polytopic membrane protein that functions as an ER cholesterol sensor to control the cellular cholesterol content ${ }^{15-20}$. When the ER cholesterol level is low, SCAP escorts the membrane-bound transcription factors SREBPs into COPII vesicles for their export from the ER to the Golgi complex. There, SREBPs are cleaved by proteases, allowing their transcriptionally active domain to enter the nucleus and promote expression of genes involved in cholesterol synthesis and uptake. Conversely, when cholesterol in the ER membrane is abundant, cholesterol binds to SCAP and triggers conformational changes that allow SCAP to interact with the integral ER membrane protein Insig, which retains SCAP in the ER along with unprocessed SREBPs. However, it remains unclear whether SCAP has other functions under cholesterol-fed conditions, except for sequestering SREBPs at the ER.

Although the ER produces cholesterol and also receives it from the PM and other sources, ER cholesterol content is low ${ }^{21}$. This is accomplished through the export of cholesterol from the ER against the concentration gradient in both vesicular and nonvesicular manners, the latter of which occurs at membrane contact sites. In particular, 
at ER-Golgi contact sites, Golgi-associated oxysterol-binding protein (OSBP) interacts with an integral ER membrane protein named vesicle-associated membrane proteinassociated protein (VAP), to transfer cholesterol from the ER to the trans-Golgi membranes, accompanied by reciprocal transfer of phosphoinositide 4-phosphate $(\mathrm{PI} 4 \mathrm{P})^{22}$. PI4P transported to the ER is hydrolyzed by the ER-localized lipid phosphatase Sac1, which thus seems to provide a driving force for cholesterol transport ${ }^{23}$.

ER-Golgi contact sites also control the transport of ceramide from the ER to the trans-Golgi membranes. This non-vesicular transport is mediated by a complex of VAP and ceramide transfer protein (CERT), and leads to the biosynthesis of SM and diacylglycerol (DAG) by SM synthase at the trans-Golgi membranes ${ }^{24-26}$. Importantly, PI4P in the trans-Golgi membranes is crucial for both OSBP and CERT to associate with the Golgi complex ${ }^{27}$ and hence the biosynthesis and metabolism of cholesterol, SM, PI4P, and DAG are either directly or indirectly interconnected ${ }^{28}$. Of note, DAG recruits to the TGN a serine/threonine kinase, protein kinase D (PKD), which phosphorylates CERT and OSBP to release these proteins from the Golgi complex ${ }^{29,30}$. PKD kinase activity also contributes to the membrane fission reaction required for transport carrier biogenesis at the $\mathrm{TGN}^{31-33}$.

On the basis of these reports and our previous data, we proposed a model in which lipid transfer at ER-Golgi contact sites promotes the biogenesis of carriers of the TGN to the cell surface (CARTS) ${ }^{34}$, which transport selective cargoes from the TGN to the $\mathrm{PM}^{35,36}$. In this context, lipid transfer at these contact sites needs to be strictly controlled on demand for TGN-to-PM transport, but the molecular mechanisms underlying this regulation remain largely elusive. In this study, we identified SCAP as a novel Sac1interacting protein at ER-Golgi contact sites. Our data show a new function of SCAP under cholesterol-fed conditions, in which SCAP, together with SREBPs, interacts with the cholesterol/PI4P exchange machinery at ER-Golgi contact sites and facilitates the biogenesis of CARTS at the TGN in an ER cholesterol-dependent manner. 


\section{Results}

\section{Identification of SCAP as a novel component of Sac1-positive ER-Golgi contact}

sites. We previously reported that the perinuclear Sacl localization represents its presence at specialized ER subdomains that are closely apposed to the TGN membranes, most likely corresponding to ER-Golgi contact $\operatorname{sites}^{34}$, rather than at the Golgi complex 37. To identify novel components of ER-Golgi contact sites, we explored Sac1interacting proteins using a Sac1 K2A mutant, which is more highly enriched in the juxtanuclear region than the wild-type (WT) protein, as revealed by its reduced peripheral staining (Fig. 1a). This mutant has replacement of two lysines in the Cterminal COPI-interacting motif with alanines. While the di-lysine COPI-interacting motif is used for retrograde transport from the cis-Golgi to the $\mathrm{ER}^{38}$, some ER integral membrane proteins, including Bap31, also utilize this signal for their movement along the ER from the cell center to the periphery ${ }^{39,40}$. The deconvolved, enhanced contrast, and increased resolution images of cells treated with 25-hydroxycholesterol (25-HC), a reagent that binds to OSBP and promotes the targeting of the VAP/OSBP complex to ER-Golgi contact sites ${ }^{22,41,42}$, showed good overlap between the green fluorescent protein (GFP)-Sac1 K2A signal and that of the integral ER membrane protein VAP-A, and also the close apposition, but not full overlap, of GFP-Sac1 K2A to the TGN marker TGN46, similarly to WT (Fig. 1b). Consistently, the continuity of GFP-Sac1 K2Apositive juxtanuclear membrane compartments with the peripheral ER membrane was demonstrated by inverse fluorescence recovery after photobleaching (iFRAP) (Fig. 1c): photobleaching of the peripheral area immediately decreased the perinuclear signals of GFP-Sac1 WT and K2A, but not of the Golgi resident protein N-acetylglucosaminyl transferase I-GFP (negative control). These results suggest that the Sac1 K2A mutant is prominently localized to ER-Golgi contact sites and validate the use of this mutant as a bait to identify interacting proteins at the contact sites.

Immunoprecipitation of FLAG-tagged Sac1 K2A followed by mass spectrometric analysis revealed multiple interactors of FLAG-Sac1 K2A (Fig. 1d, lane 2) with decreased binding of COPI coat proteins, which are major interactors for FLAG-Sac1 WT (Fig. 1d, lane 1, asterisks). The identified Sac1 K2A-interacting proteins include 
known components of ER-Golgi contact sites such as VAP-A, VAP-B, and OSBP, together with proteins with unknown functions at the contact sites, including SCAP (Fig. 1e). In here, we focus on SCAP because, although it had been previously shown to interact with VAP-A and VAP-B, the physiological role of this interaction remains unexplored $^{43}$.

\section{ER-localized SCAP interacts through Sac1 with the VAP-A/OSBP complex at ER-}

Golgi contact sites. In order to confirm that SCAP is a bona fide component of ERGolgi contact sites, the interactions of FLAG-tagged hamster SCAP with VAP-A, OSBP, and Sac1 were examined by immunoprecipitation (Fig. 2a). Endogenous Sac1 and VAP-A were clearly coprecipitated with FLAG-SCAP, but not with the FLAG vector control (Fig. 2a, lanes 7 and 9). When Myc-OSBP was coexpressed, this protein was coprecipitated with FLAG-SCAP and the interaction of FLAG-SCAP with VAPA, but not with Sac1, was enhanced (Fig. 2a, lanes 9 and 10). As negative controls, we showed that an integral ER membrane protein, reticulon-4B (RTN-4B), and another lipid transfer protein, CERT, were not coprecipitated with FLAG-SCAP (Fig. 2b).

Next, we asked whether SCAP interacts with the VAP-A/OSBP complex via Sac1. Our previous work showed that Sac1 interacts with VAP-A via OSBP ${ }^{34}$. We used two OSBP mutants, FF/AA and PH-FFAT, that are defective in binding to VAP and Sac1, respectively (Ref $\mathrm{R}^{44,45}$ and Supplementary Fig. 1a,b). In cells expressing Myc-OSBP FF/AA, this protein, but not VAP-A, was found to interact with FLAG-SCAP (Fig 2a, lane 11), suggesting that VAP-A is not needed for the interaction of SCAP with OSBP. By contrast, Myc-OSBP PH-FFAT was not coprecipitated with FLAG-SCAP (Fig 2a, lane 12). Although previous work revealed that Myc-OSBP PH-FFAT fixes and expands ER-Golgi contact sites through its stable interaction with VAP-A ${ }^{22}$, it inhibited the interaction of FLAG-Sac1 with VAP-A (Supplementary Fig. 1, lane 12). MycOSBP PH-FFAT defective in Sac1 binding also greatly reduced the interaction of FLAG-SCAP with VAP-A (Fig. 2a, lane 12), emphasizing the requirement of Sac1 for the interaction of SCAP with the VAP-A/OSBP complex. Similar results were obtained

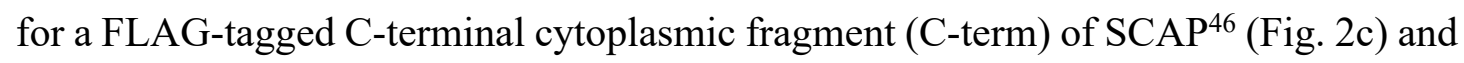


the specific interaction between FLAG-SCAP and Sac1 was also detected in HeLa cells stably expressing FLAG-SCAP at a level of about four times the level of endogenous SCAP (Supplementary Fig. 1c,d). These results exclude the possibility of membrane aggregation or co-sedimentation with FLAG-SCAP. The specific but relatively weak interactions of SCAP with the cholesterol/PI4P exchange machinery most likely reflect transient interactions among these components, consistent with the underlying association-dissociation dynamics of ER-Golgi contacts that are crucial for their function $^{22,34}$.

To corroborate that ER-localized SCAP, but not Golgi-localized SCAP, can form complexes with VAP-A, OSBP, and Sac1 at ER-Golgi contact sites, we used a SCAP mutant with replacement of aspartic acid 451 and leucine 452 with alanines at the MELADL motif in loop 6 (D451A/L452A), which is defective in COPII binding and therefore cannot exit the ER in COPII-coated vesicles ${ }^{47}$. FLAG-SCAP D451A/L452A showed interactions with VAP-A, OSBP, and Sac1, analogous to FLAG-SCAP WT (Fig. 2d). We next visualized the interactions of SCAP with these proteins in intact cells by using bimolecular fluorescence complementation (BiFC). As shown previously ${ }^{48,49}$, a BiFC signal derived from the Vn-OSBP/Vc-VAP-A interaction was detected at the perinuclear region representing ER-Golgi contact sites (Supplementary Fig. 2c, top row), whereas no signal was detected when only $\mathrm{Vn}$ - or Vc-fused constructs were individually expressed (Supplementary Fig. 2a,b). This signal was enhanced by $25-\mathrm{HC}$ treatment (Supplementary Fig. 2c, middle row). Similar results were obtained with the combination of Vn-OSBP/Vc-Sac1 (Supplementary Fig. 2d). The Vn-SCAP/Vc-Sac1 and Vn-SCAP/Vc-VAP-A interactions were also observed upon coexpression of MycOSBP, and these interactions were enhanced by 25-HC treatment (Fig. 2e,f,g,h), similar to the Vn-OSBP/Vc-VAP-A and Vn-OSBP/Vc-Sac1 interactions (Supplementary Fig. 2c,d). Upon 25-HC treatment, the BiFC signal derived from the Vn-SCAP and Vc-Sac1 or Vc-VAP-A interaction was in close apposition to the TGN46 signal, confirming that these interactions occur at ER-Golgi contact sites (Fig. 2i,j).

To further substantiate that the perinuclear Vn-SCAP/Vc-Sac1 and Vn-SCAP/VcVAP-A BiFC signals represent the interaction of ER-localized SCAP, but not of Golgi- 
localized SCAP, we examined the effect of cholesterol depletion. As reported previously ${ }^{50}$, cholesterol depletion using lipoprotein-deficient serum and 2hydroxypropyl- $\beta$-cyclodextrin caused redistribution of a part of the SCAP pool from the ER to the cis/medial Golgi membranes (Supplementary Fig. 3a,b) accompanied by SREBP2 cleavage (Supplementary Fig. 3c). In contrast to 25-HC treatment, cholesterol depletion did not enhance either the Vn-SCAP/Vc-Sac1 or Vn-SCAP/Vc-VAP-A interaction (Fig. 2e,f, bottom row, and Fig 2g,h). Altogether, these results suggest that ER-localized SCAP preferentially interacts with VAP-A, OSBP, and Sac1 at ER-Golgi contact sites.

\section{SCAP is important for PI4P turnover and VAP-A/OSBP complex distribution at}

ER-Golgi contact sites. The finding that SCAP forms complexes with VAP-A, OSBP, and Sac1 at ER-Golgi contact sites prompted us to examine whether SCAP is involved in the counter-transport of ER cholesterol and Golgi PI4P at ER-Golgi contact sites. To address this issue, we performed knockdown of SCAP in HeLa cells. By using siRNA, the expression of SCAP was decreased to $\sim 20 \%$ of the control level (Fig. 3a). In response to cholesterol deprivation, the SCAP-SREBP pathway stimulates transcription of genes responsible for cholesterol synthesis and uptake, such as 3-hydroxy-3methylglutaryl coenzyme A reductase (HMGR) and low-density lipoprotein receptor $(\text { LDLR })^{16,51}$. However, under cholesterol-fed conditions -that is, culturing of cells in normal medium supplemented with fetal calf serum (FCS) as a source of sterols-, SCAP knockdown did not reduce the transcription of either HMGR or LDLR genes, but rather slightly increased the LDLR mRNA levels (Fig. 3b). Consistently, no significant change in the total cholesterol level was observed upon SCAP knockdown (Fig. 3c). Similar results were obtained for a HeLa stable cell line (shSCAP cells) with reduced expression of SCAP ( $\sim 10 \%$ of that in parental HeLa cells), but not of HMGR and LDLR (Fig. 3d,e,f).

Staining of parental HeLa and shSCAP cells with the fluorescent cholesterol probe filipin showed no significant difference in the intracellular distribution of cholesterol between these cell lines (Fig. 4a). Although a part of the perinuclear signal of filipin 
overlapped with that of a TGN marker, Golgin-97, most of the signal was thought to be derived from other membranous structures, as shown previously ${ }^{21}$, and filipin does not seem to have sufficient sensitivity to detect low levels of cholesterol in ER and Golgi membranes.

Because of the lack of cholesterol probes with enough sensitivity for our purposes, we focused on monitoring PI4P level in SCAP knockdown cells. When PI4P was visualized with a specific antibody, the fluorescence signal in shSCAP cells was significantly increased as compared to that in the parental HeLa cells, especially in the juxtanuclear region that partially overlapped with membranes positive for the TGN markers sialyltransferase and Golgin-97 (Fig. 4b,c). Similar phenotypes were observed upon VAP-A/B or CERT/OSBP double knockdown, as well as in Sac1 knockdown cells (Supplementary Fig. 4a). Taken together, our data suggest that SCAP regulates the TGN PI4P levels by controlling the turnover of PI4P at ER-Golgi contact sites.

Next, we evaluated the effect of SCAP knockdown on the formation of the VAPA/OSBP complex by using the BiFC approach. A stable cell line coexpressing VnOSBP and Vc-VAP-A showed a BiFC signal in the juxtanuclear region, which was enhanced by $25-\mathrm{HC}$ treatment (Supplementary Fig. 4b). When SCAP was knocked down by siRNA, $\sim 34 \%$ of cells showed a bright BiFC signal in the peripheral region, in addition to the signal at the juxtanuclear Golgi region, and the number of cells with this phenotype increased to $\sim 62 \%$ upon 25 -HC treatment (Fig. 4 d). As the VAP/OSBP complex has been reported to exist not only at ER-Golgi contact sites, but also at ERendosome contact sites $^{52}$, we compared the localization of the peripheral BiFC signal with that of the late endosomal marker CD63. The close apposition of CD63 but not of TGN46 to the peripheral BiFC signal was observed in SCAP knockdown cells (Fig. 4e), indicating that SCAP is required to prevent the redistribution of the VAP-A/OSBP complex to ER-endosome contact sites and to maintain it at ER-Golgi contact sites.

SCAP is required for the biogenesis of CARTS at the TGN. We previously reported that perturbation of lipid transfer complexes at ER-Golgi contact sites inhibits the biogenesis of CARTS at the TGN ${ }^{34}$. We now visualized CARTS in Vn-OSBP/Vc-VAP- 
A expressing cells treated with $25-\mathrm{HC}$ by staining a CARTS specific cargo, pancreatic adenocarcinoma up-regulated factor (PAUF) ${ }^{35}$. Deconvolved images showed that putative nascent CARTS were located in the close vicinity of BiFC-positive perinuclear region (Fig. 5a), suggesting that CARTS form at sites immediately adjacent to the VAPA/OSBP-containing ER-Golgi contact sites. Next, we investigated whether SCAP is required for CARTS-mediated protein secretion. In SCAP knockdown cells, the secretion of PAUF-MycHis was reduced to $\sim 50 \%$ of that in control cells (Fig. 5b). Moreover, the small amount of PAUF-MycHis secreted by SCAP knockdown cells was detected as a smeared band, suggesting a defect in PAUF processing, as previously observed in cells depleted of other ER-Golgi contact site components ${ }^{34}$.

The effect of SCAP knockdown on the biogenesis of CARTS was assessed with an inducible CARTS formation assay, where synchronized transport of PAUF from the ER is carried out by using a reverse dimerization system involving $\mathrm{D} / \mathrm{D}$ solubilizerinduced disassembly of FM4 domains ${ }^{53}$. The chimera protein mKate2-FM4-PAUF initially retained in the ER was first exported from this organelle and accumulated in the Golgi membranes by $\mathrm{D} / \mathrm{D}$ solubilizer treatment at $20^{\circ} \mathrm{C}$ for $45 \mathrm{~min}$, after which the temperature was shifted to $37^{\circ} \mathrm{C}$ to induce the formation of mKate2-FM4-PAUFcontaining CARTS at the TGN. Live cell imaging showed that a large number of CARTS were formed at the TGN membranes and dispersed throughout the cytoplasm (Supplementary Video 1). Next, mKate2-FM4-PAUF was expressed in control and SCAP knockdown cells. At 15 min after the temperature shift to $37^{\circ} \mathrm{C}$, the average number of CARTS in SCAP knockdown cells was $\sim 20 \%$ of that in control cells (Fig. $5 c)$.

In our model, lipid transfer at ER-Golgi contact sites is thought to promote CARTS biogenesis through organization of cholesterol- and SM-enriched nanodomains at the $\mathrm{TGN}^{34}$. We therefore examined the effect of SCAP knockdown on transport of glycosylphosphatidyl inositol (GPI)-anchored protein, which has been reported to associate with such lipid nanodomains ${ }^{1,54,11,55}$. Synchronized transport of mKate2FM4-GPI from the ER to the PM was initiated by addition of the D/D solubilizer, as previously reported ${ }^{11}$. SCAP knockdown did not affect transport of mKate2-FM4-GPI 
from the ER ( $0 \mathrm{~min})$ to the Golgi complex $(30 \mathrm{~min})$. Nevertheless, at $60 \mathrm{~min}$ after transport initiation, the amount of mKate2-FM4-GPI that had reached the cell surface was significantly decreased in SCAP knockdown cells (Fig. 6a). At 90 min most of the protein had been transported to the PM in control cells, but in $\sim 40 \%$ of SCAP knockdown cells, the protein was still localized to the TGN (Fig. 6a,b). Similar results were obtained with knockdown of VAP-A/B, CERT/OSBP, or, to a lesser extent, OSBP (Fig. 6a and Supplementary Fig. 5a). In addition to these results, overexpression of OSBP PH-FFAT, which immobilizes ER-Golgi contact sites and inhibits CARTS biogenesis $^{34}$, strongly inhibited mKate2-FM4-GPI export from the TGN (Supplementary Fig. 5b).

We tested if CARTS are the carriers that transport mKate2-FM4-GPI from the TGN to the cell surface. When mKate2-FM4-GPI and PAUF-MycHis were coexpressed in cells, we observed that mKate2-FM4-GPI was included in PAUFMycHis-positive CARTS, although its fluorescence signal was relatively low (Fig. 6c). Consistent with our previous finding that CARTS biogenesis requires PKD-mediated membrane fission at the $\mathrm{TGN}^{35}$, export of mKate2-FM4-GPI from the TGN was inhibited by expression of a dominant-negative kinase-dead mutant of PKD2 and even at 90 min after transport initiation, the protein was included in TGN-derived tubules, most likely corresponding to fission-defective precursors of transport carriers, such as CARTS (Fig. 6d). Previous work revealed that a non-toxic SM reporter protein, EQSM is enriched in transport carries containing mKate2-FM4-GPI ${ }^{11}$. When EQ-SM and PAUF-MycHis were coexpressed in cells, $\sim 80 \%$ of PAUF-MycHis-positive CARTS contained EQ-SM (Fig. 6e). This result fits well with the former report that $86 \pm 5 \%$ of mKate2-FM4-GPI-positive exocytic vesicles contain EQ-SM ${ }^{11}$. Taken together, these results strongly suggest that SCAP promotes the biogenesis of CARTS, which are enriched in cholesterol and SM.

\section{SCAP regulates CARTS biogenesis in a cholesterol-dependent manner. SCAP}

directly binds cholesterol and plays a pivotal role in cholesterol homeostasis as a cholesterol sensor in the ER membrane ${ }^{20,56,57}$. Does SCAP regulate CARTS biogenesis 
by sensing the ER cholesterol level? Previous mutagenesis analysis of SCAP in Chinese hamster ovary $(\mathrm{CHO})$ cells revealed that several amino acid residues are critical for the conformational change induced by cholesterol binding ${ }^{20}$. Specifically, replacement of tyrosine 234 in the cholesterol-binding loop1 region of SCAP (Fig. 7a) with alanine (Y234A) abolishes binding of loop1 to loop7, and thus this mutant binds to Insig even in the absence of cholesterol ${ }^{57}$. By contrast, a mutant with replacement of tyrosine 298 in the transmembrane sterol-sensing domain (Fig. 7a) with cysteine (Y298C) is resistant to the cholesterol-induced conformational change and shows no Insig binding even in the presence of sterols ${ }^{43,58-61}$. We established different shSCAP cells, each of which stably expressing hamster SCAP WT, Y234A, or Y298C. Western blotting of cell lysates with an antibody specific to hamster SCAP, and one recognizing both human and hamster SCAP indicated that Y234A was less expressed than WT and Y298C, but it was still expressed at a considerably higher level than the endogenous protein in parental HeLa cells (Fig. 7b). Immunofluorescent staining with an anti-hamster SCAP antibody showed reticular distributions of WT, Y234A, and Y298C in the ER, under cholesterol-fed conditions (Supplementary Fig. 3d, upper row). In a previous work, digestion of $N$-linked carbohydrates of SCAP by endoglycosidase (endo) H was shown to indicate that SCAP Y298C is redistributed to the Golgi complex even in the presence of sterols ${ }^{58}$. However, at the level of immunofluorescence microscopy, the signal of Y298C in the Golgi complex was not obvious, because most of the protein was present in the juxtanuclear ER (Supplementary Fig. 3d, upper right panel). Perhaps, a minor fraction of the Y298C pool is transported to the Golgi complex and acquires endo $\mathrm{H}$ resistance in the presence of sterols. When cholesterol was depleted from cells, fractions of the WT and Y298C pools were redistributed from the ER to the Golgi complex, but Y234A remained in the ER (Supplementary Fig. 3d, lower row), in agreement with previous reports ${ }^{57,58,60}$.

By using these cell lines, we first examined the effect that the expression of the respective hamster SCAP proteins has on the turnover of PI4P. Our results showed that expression of WT, but not Y234A or Y298C, reversed the accumulation of PI4P at the TGN (Fig. 7c). We next examined their effects on CARTS biogenesis. Expression of 
SCAP WT caused the recovery of the number of CARTS from SCAP knockdown, but neither Y234A nor Y298C showed such an effect (Fig. 7d). These results suggest that the sterol-sensing ability of SCAP is required for CARTS biogenesis, tightly linked to the capacity of SCAP to regulate PI4P turnover at ER-Golgi contact sites.

SCAP/SREBP complex functions in CARTS biogenesis. SCAP and SREBPs are known to form a stable complex. Consistent with previous reports ${ }^{62-64}$, expression levels of SREBP1 and SREBP2 were also significantly decreased in shSCAP cells (Fig. 8a, lane 2). Importantly, while expression of not only SCAP WT, but also Y234A or Y298C recovered expression of SREBPs (Fig. 8a, lanes 3-5), these mutants were not able to rescue the defects in PI4P turnover and CARTS biogenesis (Fig. 7c,d). These results suggest that the sterol-sensing ability of SCAP, and not the presence of SREBPs, is the dominant factor by which SCAP controls PI4P turnover and CARTS biogenesis. Immunoprecipitation of FLAG-SREBP1a and FLAG-SREBP2 showed their interactions with Myc-OSBP and endogenous Sac1 and VAP-A (Fig. 8b). Our observation that FLAG-SREBPs show a higher affinity for GFP-SCAP than for components of the cholesterol/PI4P exchange machinery (Fig. 8c), suggests that SCAP/SREBPs form a stable complex that only transiently interacts with the lipid exchange machinery at ER-Golgi contact sites. Finally, we tested whether knockdown of SREBPs affects CARTS biogenesis. The expression levels of SREBP1 and SREBP2 was significantly decreased by using siRNA targeting common sequence of two alternative splicing isoforms of SREBP1 (1a and 1c) and SREBP2, respectively, without affecting expression of SCAP (Fig. 8d, lanes 1-3). Since a mixture of these two siRNAs did not reduce SREBP1 and SREBP2 at the same time (Fig. 8d, lane 4), the effect on CARTS formation of either SREBP1 or SREBP2 knockdown was examined. Our results showed that the average number of CARTS was decreased to $\sim 71 \%$ and $\sim 36 \%$ of the control levels in SREBP1 and SREBP2 knockdown cells, respectively (Fig. 8e). 


\section{Discussion}

SCAP was discovered in 1996 as an ER protein whose mutation conferred, on CHO cells, resistance to 25-HC, an oxygenated cholesterol derivative that suppresses SREBP processing and thereby blocks cholesterol synthesis, but cannot replace cholesterol for cell viability ${ }^{15}$. Posterior studies demonstrated that SCAP senses the ER cholesterol content and, in response to cholesterol deficiency, escorts SREBPs from the ER to the Golgi complex for their cleavage-mediated activation ${ }^{16-20}$.

In the present study, we demonstrated a new function of SCAP under cholesterolfed conditions. Based on our data, we propose a model whereby SCAP interacts with VAP/OSBP at ER-Golgi contact sites via Sac1 and regulates counter-transport of cholesterol and PI4P in an ER cholesterol-dependent manner (Fig. 9). This idea is supported by our finding that SCAP knockdown causes accumulation of PI4P at the TGN (Fig. 4b,c), a hallmark of the impairment of cholesterol/PI4P exchange between the ER and the trans-Golgi membranes. SCAP appears to contribute to the efficient establishment of ER-Golgi contact sites because its knockdown caused partial redistribution of the VAP/OSBP complex to ER-endosome contact sites (Fig. 4d,e). At the trans-Golgi membranes, cholesterol and SM organize lipid nanodomains, which can function as a platform for molecular machineries responsible for processing and sorting of cargoes, including GPI-anchored proteins (Fig. 9, right panel). In parallel, DAG, which is synthesized together with SM from ceramide and phosphatidylcholine, recruits PKD for membrane fission, leading to CARTS biogenesis. Intriguingly, our finding of putative nascent CARTS in the close vicinity to ER-Golgi contact sites (Fig. 5a) suggests a possible role of the ER contacts in determining the position for membrane fission, analogous to their important role in mitochondrial and endosomal fission $^{65,66}$.

Cytoplasmic coats generally provide a means to coordinate signal-mediated cargo sorting with carrier budding and membrane fission. However, most TGN-derived transport carriers, including CARTS, lack cytoplasmic coats and therefore such molecular mechanisms remain largely elusive for these transport carriers ${ }^{35,67}$. A recent paper reported that $\mathrm{Ca}^{2+}$-dependent and oligomerization-driven cargo sorting, which is 
mediated by the SPCA1 $\mathrm{Ca}^{2+}$ pump and the secreted $\mathrm{Ca}^{2+}$ binding protein $\mathrm{Cab} 45$, is coupled to local $\mathrm{SM}$ synthesis at the $\mathrm{TGN}^{14}$. The finding that sorting of lysozyme $\mathrm{C}$, one of the secretory cargoes in CARTS ${ }^{35}$, is controlled by this mechanism strongly supports our model.

Our data showed that neither the SCAP Y234A nor Y298C mutants, which reflect different conformations in the context of Insig binding, are competent for PI4P turnover and CARTS biogenesis (Fig. 7c,d). These findings imply that conformational switching that reflects cholesterol-free and -bound states is important. Considering that the ER contains low levels of cholesterol, it is tempting to speculate that SCAP functions in collecting cholesterol in the ER membrane to present it to cytosolic OSBP for efficient cholesterol transfer at ER-Golgi contact sites. It is of note that SCAP knockdown dramatically altered the distribution of the VAP/OSBP complex in the presence of 25$\mathrm{HC}$ (Fig. 4d). As $25-\mathrm{HC}$ directly binds to $\mathrm{OSBP}^{41,42}$, but indirectly to SCAP via Insig $^{68,69}$, this finding reveals the possibility that SCAP functions at ER-Golgi contact sites as a complex with Insig, as well as SREBPs, supported by a previous finding that Insig interacts with VAP-A and VAP-B ${ }^{70}$.

In conclusion, our findings reveal a new role of SCAP under cholesterol-fed conditions and provide insights into the regulatory mechanisms for lipid transfer at ERGolgi contact sites for transport carrier biogenesis at the TGN. 


\section{Materials and methods}

\section{Antibodies and reagents}

Monoclonal antibodies were procured as follows: FLAG and alpha-tubulin were purchased from Sigma-Aldrich; Myc, hamster SCAP (clone: 9D5), and HMGR from Santa Cruz Biotechnology; SREBP1 from EMD Millipore; Penta-His from QIAGEN; CD63 (clone H5C6) from the Developmental Studies Hybridoma Bank; and PI4P from Echelon Biosciences. Polyclonal antibodies were procured as follows: HA, Nogo (RTN-4B), SCAP, and GST from Santa Cruz Biotechnology; TGN46 (sheep IgG) from AbD Serotec; GFP from Thermo Fisher Scientific; SREBP2, LDLR, and Golgin-97 from Abcam; and Myc from Cell Signaling Technology. To raise rabbit polyclonal antibodies against TGN46 and Sac1, GST-tagged fragments of human TGN46 (aa 1365) and Sac1 (aa 1-55), respectively, were expressed in Escherichia coli, purified, and used as antigens. These antibodies were isolated by affinity chromatography on antigen-coupled beads. An anti-VAP-A and anti-Bap31 polyclonal antibodies were produced as described previously ${ }^{34,39}$. An anti-Mannosidase II polyclonal antibody was provided by V. Malhotra (Centre for Genomic Regulation, Barcelona, Spain). D/D solubilizer were purchased from Clontech. Methyl- $\beta$-cyclodextrin and 2hydroxypropyl- $\beta$-cyclodextrin were purchased from Wako Chemicals. Filipin and 25HC were purchased from Sigma-Aldrich.

\section{Plasmids}

The plasmids encoding PAUF-MycHis, N-acetylglucosaminyl transferase I-GFP, Myc$\mathrm{OSBP}^{71}$, and HA-CERT ${ }^{72}$ were kindly donated by S. S. Koh (Korea Research Institute of Bioscience and Biotechnology, Daejeon, Korea), N. Nakamura (Kyoto Sangyo University, Kyoto, Japan), H. Arai (University of Tokyo, Tokyo, Japan), K. Hanada (National Institute of Infectious Diseases, Tokyo, Japan), respectively. The plasmids encoding mKate2-FM4-GPI and EQ-SM (tagged with oxGFP) were generous gifts from C. G. Burd (Yale School of Medicine, New Haven, CT, USA). The plasmids encoding the GFP-Sac1 WT and K2A mutant were generous gifts from P. Mayinger (Oregon Health and Science University, Portland, OR, USA). The plasmids encoding 
FLAG-SREBP1a and FLAG-SREBP2 were generous gifts from D. M. Sabatini (Whitehead Institute for Biomedical Research, Cambridge, MA, USA) (Addgene

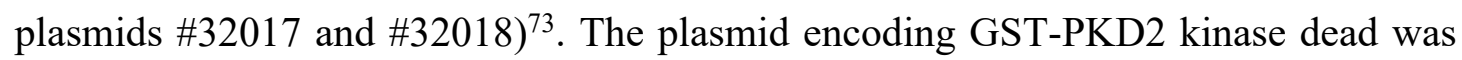
provided by V. Malhotra (Centre for Genomic Regulation, Barcelona, Spain). The cDNAs encoding hamster SCAP WT and C-term (aa 732-1276) were amplified by PCR from pCMV-GFP-SCAP, a generous gift from P. Espenshade (Johns Hopkins University School of Medicine, Baltimore, MD, USA), and inserted into pFLAGCMV-6c to express the protein with an N-terminal FLAG. For expression of FLAGSCAP D451A/L452A, point mutations were introduced into pFLAG-SCAP by PCR using primers designed for replacing aspartic acid 451 and leucine 452 with alanines. The cDNA encoding hamster SCAP or rabbit OSBP (WT or FF/AA) were inserted into pCI-IRES-Bsr in combination with one encoding the N-terminal fragment of Venus (Vn) (aa 1-172). The cDNA encoding human Sac1 or VAP-A was inserted into pCIIRES-Bsr in combination with one encoding the C-terminal fragment of Venus ( $\mathrm{Vc})$ (aa 154-238). For establishment of stable cell lines, the cDNAs encoding Vn-OSBP and Vc-VAP-A were inserted into the pMXs-IRES-Puro and pCX4-IRES-Bsr retroviral vectors, respectively, and hamster SCAP with or without N-terminal FLAG or GFP was inserted into pCX4-IRES-Bsr. For expression of SCAP Y234A or Y298C, each point mutation was introduced into pCX4-SCAP-IRES-Bsr by PCR using primers designed for replacing tyrosine 234 or 298 with alanine. For expression of mKate2-FM4-PAUF, the cDNA encoding GPI in the plasmid for mKate2-FM4-GPI was replaced with one encoding human PAUF (aa 43-197). The cDNA encoding human sialyltransferase (aa 1-69) was inserted into a pcDNA3-based plasmid encoding mRFP to express the protein with a C-terminal mRFP. Plasmids for Myc-OSBP FF/AA and PH-FFAT were described previously ${ }^{34}$. For establishment of shSCAP HeLa cells, the Mission shRNA plasmid (TRCN0000289279) was purchased from Sigma-Aldrich.

\section{siRNA and shRNA}

The targeting sequences of siRNA and shRNA were as follows:

Control (GL2 luciferase): 5'-AACGTACGCGGAATACTTCGA-3' 
SCAP (siRNA): 5'-AACCTCCTGGCAGTAGATGTA-3'

SCAP (shRNA): 5'- GCTCTGGTGTTCTTGGACAAA-3'

VAP-A: 5'-AACTAATGGAAGAGTGTAAAA-3'

VAP-B: 5'-AAGAAGGTTATGGAAGAATGT-3'

OSBP: 5'-AATACTGGGAGTGTAAAGAAA-3'

CERT: 5'-AAGAACAGAGGAAGCATATAA-3'

Sac1: 5'-AACTGATATTCAGTTACAAGA-3'

SREBP1: 5'-GAGGCAAAGCTGAATAAATCT-3'

SREBP2: 5'-CAGGCTTTGAAGACGAAGCTA-3'

\section{Cell culture and transfection}

HeLa and HEK 293T cells were grown in DMEM supplemented with 10\% FCS. PLATA packaging cells were grown in DMEM supplemented with $10 \% \mathrm{FCS}, 10 \mu \mathrm{g} / \mathrm{ml}$ blasticidin S, and $1 \mu \mathrm{g} / \mathrm{ml}$ puromycin. Plasmid and siRNA transfection into HeLa cells were carried out using X-tremeGENE 9 DNA transfection reagent (Roche) and Oligofectamine reagent (Invitrogen), respectively, according to the manufacturers' protocols. Plasmid transfection into HEK 293T cells was carried out using polyethylenimine (Polysciences) or Lipofectamine 2000 transfection reagent (Invitrogen), according to the manufacturers' protocols.

\section{Establishment of stable cell lines}

For establishment of HeLa cells stably expressing FLAG- or GFP-SCAP, PLAT-A packaging cells were transfected with pCX4-FLAG- or GFP-SCAP-IRES-Bsr, and 48 $\mathrm{h}$ later, the medium containing retrovirus was collected and used for infection of HeLa cells. Selection of HeLa cells stably expressing FLAG- or GFP-SCAP was performed with $10 \mu \mathrm{g} / \mathrm{ml}$ blasticidin S. A HeLa stable cell line coexpressing Vn-OSBP and VcVAP-A was established in a similar manner with pMXs-Vn-OSBP-IRES-Puro and pCX4-Vc-VAP-A-IRES-Bsr by selecting cells with $1 \mu \mathrm{g} / \mathrm{ml}$ puromycin and $10 \mu \mathrm{g} / \mathrm{ml}$ blasticidin S. For establishment of shSCAP HeLa cells, HEK 293T cells were cotransfected with the Mission shRNA plasmid (TRCN0000289279), pMDLg/pRRE, 
pRSV-Rev, and pCMV-VSVG. After $48 \mathrm{~h}$, the medium containing lentivirus was collected and used for infection of HeLa cells. Selection of shSCAP HeLa cells was performed with $1 \mu \mathrm{g} / \mathrm{ml}$ puromycin. Establishment of shSCAP HeLa cells stably expressing hamster SCAP WT, Y234A, or Y298C was performed as described for GFP-SCAP HeLa cells with pCX4-SCAP (WT, Y234A, or Y298C)-IRES-Bsr. All the stable cell lines were obtained without single-cell cloning.

\section{Immunofluorescence microscopy}

HeLa cells were fixed with 4\% paraformaldehyde (PFA) in phosphate-buffered saline (PBS) at room temperature for $20 \mathrm{~min}$, permeabilized with $0.2 \%$ Triton X-100 in PBS for $30 \mathrm{~min}$, and then blocked with $2 \%$ bovine serum albumin (BSA) in PBS for $30 \mathrm{~min}$. The cells were labeled with the indicated primary antibodies and secondary antibodies conjugated to Alexa Fluor 488, 594, or 633 in the blocking buffer. The samples were analyzed with an Olympus Fluoview FV1000 or FV1200 confocal microscope with a UPLSAPO 60x O NA 1.35 objective and FV10-ASW software. Image processing and measurement of fluorescence intensity were performed with ImageJ software (National Institutes of Health). Vesicular structures containing EQ-SM and/or PAUF-MycHis were detected using the plugin ComDet (https://github.com/ekatrukha/ComDet).

\section{Image deconvolution}

Deconvolution processing was performed with Huygens Professional version 18.04 (Scientific Volume Imaging, The Netherlands, http://svi.nl). For that, a theoretical point-spread function (PSF) was automatically computed based on the microscope and image acquisition parameters. The deconvolution process was numerically performed using the Classic Maximum Likelihood Estimation (CMLE) algorithm. In brief, this algorithm assumes that the photon noise is Poisson-distributed, and the likelihood of an estimate of the actual image given the computed PSF and the acquired image is iteratively optimized until either a quality factor or a maximum number of iterations is reached. In our deconvolutions, we used a quality factor equal to 0.001 and a maximum 50 iterations. The signal-to-noise ratio (SNR) for each acquired image was computed 
based on three line profiles going through regions of background signals towards regions of positive, actual signals. Typically, SNR were of the order of 7-20 for the different analyzed images.

\section{Filipin staining}

Filipin staining was performed as described previously ${ }^{74}$. In brief, HeLa cells were treated with $10 \mathrm{mM}$ Methyl- $\beta$-cyclodextrin at $37^{\circ} \mathrm{C}$ for $30 \mathrm{~min}$ and fixed with $4 \% \mathrm{PFA}$ at room temperature for $10 \mathrm{~min}$. The cells were incubated with $0.1 \mathrm{mg} / \mathrm{ml}$ filipin in PBS at room temperature for $30 \mathrm{~min}$. After washing with PBS, the cells were blocked with 1\% BSA in PBS for 30 min and labeled with anti-Golgin-97 antibody and a secondary antibody conjugated to Alexa Fluor 594 in the blocking buffer. After re-incubation with $0.1 \mathrm{mg} / \mathrm{ml}$ filipin at room temperature for $30 \mathrm{~min}$, the cells were washed with PBS and then mounted on a microscope slide. The samples were analyzed by fluorescence microscopy as described above.

\section{PI4P staining}

PI4P staining was performed as described previously ${ }^{13,75}$. In brief, HeLa cells were fixed with $2 \%$ PFA at room temperature for $15 \mathrm{~min}$, followed by washing with PBS containing $50 \mathrm{mM} \mathrm{NH}_{4} \mathrm{Cl}$. The cells were then permeabilized with $20 \mu \mathrm{M}$ digitonin in buffer A (20 mM PIPES, pH 6.8, $137 \mathrm{mM} \mathrm{NaCl}, 2.7 \mathrm{mM} \mathrm{KCl})$. After removal of digitonin by washing with buffer A, the cells were blocked with 5\% FCS in buffer A for $45 \mathrm{~min}$. The cells were labeled with anti-PI4P and anti-Golgin-97 antibodies, followed by secondary antibodies conjugated to Alexa Fluor 488 and 594, respectively, in the blocking buffer. After post-fixation with 2\% PFA for $5 \mathrm{~min}$, the cells were washed with PBS containing $50 \mathrm{mM} \mathrm{NH}_{4} \mathrm{Cl}$ and with water, and then mounted on a microscope slide. The samples were analyzed by fluorescence microscopy as described above.

\section{Quantification of Golgi PI4P}


To quantify the intensity of the PI4P signal at the Golgi complex from our immunofluorescence microscopy images, we used the ImageJ distribution Fiji ${ }^{76}$ and a custom-made macro, following the method described in Ref ${ }^{77}$. First, we generated a binary mask for the Golgi/TGN area using the signal of the marker protein Golgin-97 after 1-pixel radius Gaussian blur filtering. Then, the mean intensities of the PI4P and Golgin-97 channels in the Golgi/TGN mask areas were background subtracted and measured separately for each individual cell. Finally, the measured Golgi (mask) PI4P levels were normalized to the Golgi (mask) Golgin-97 levels per each cell.

\section{Quantification of perinuclear BiFC signal}

To quantify the perinuclear BiFC signal from our fluorescence microscopy images, we used the Image J distribution Fiji and a custom-made macro. First, we generated a binary mask for the perinuclear area using the signal of the TGN marker protein TGN46 after 1-pixel radius Gaussian blur filtering. Finally, the total intensity of the BiFC channel in the perinuclear mask area was measured separately for each individual cell after background subtraction.

\section{iFRAP}

HeLa cells expressing GFP-Sac1 WT, K2A, or N-acetylglucosaminyl transferase IGFP in Opti-MEM were cultured in $5 \% \mathrm{CO}_{2}$ at $37^{\circ} \mathrm{C}$ during live-cell imaging. The cells were subjected to bleaching with high laser intensity (473 nm laser) for $15 \mathrm{~s}$, followed by an imaging scan with a time interval between frames of $10 \mathrm{~s}$ for $\sim 4$ min by use of an Olympus Fluoview FV1000 confocal microscope with a UPLSAPO 100x O NA 1.40 objective and FV10-ASW software. Image processing and measurement of fluorescence intensity were performed with ImageJ software.

\section{Immunoprecipitation}

HEK 293 T cells were lysed in buffer B (50 mM HEPES-KOH, pH 7.4, 100 mM NaCl, $1.5 \mathrm{mM} \mathrm{MgCl} 2,1 \mathrm{mM}$ dithiothreitol, 1\% Nonidet P-40, $1 \mu \mathrm{g} / \mathrm{ml}$ leupeptin, $2 \mu \mathrm{M}$ pepstatin A, $2 \mu \mathrm{g} / \mathrm{ml}$ aprotinin, and $1 \mathrm{mM}$ phenylmethylsulfonyl fluoride). The lysates 
were centrifuged at $17,000 \times \mathrm{g}$ for $10 \mathrm{~min}$. The resulting supernatants were immunoprecipitated with an anti-FLAG M2 affinity gel (Sigma-Aldrich), and the precipitated proteins were analyzed by Western blotting with the indicated primary antibodies and secondary antibodies conjugated to horseradish peroxidase. For immunoprecipitation of FLAG-SREBPs and stably expressed FLAG-SCAP, cells were lysed in buffer B without dithiothreitol and the lysates were centrifuged at 17,000 $\times \mathrm{g}$ for $10 \mathrm{~min}$. The supernatants were further centrifuged at $100,000 \times \mathrm{g}$ for $30 \mathrm{~min}$ and the resulting supernatants were immunoprecipitated as described above. For identification of the Sac1 interacting proteins, the precipitated proteins were eluted with FLAG peptide and analyzed by silver staining. Protein bands were excised from the gel and subjected to LC-MS/MS analysis.

\section{LC-MS/MS analysis}

Each gel piece was incubated with $0.25 \mu \mathrm{g}$ trypsin in $20 \mu 1$ Tris-HCl ( $\mathrm{pH} 8.8$ ) overnight at $37^{\circ} \mathrm{C}^{78}$, and the resulting peptide mixture was analyzed with a direct nanoflow LCMS/MS system equipped with an hybrid quadrupole-orbitrap mass spectrometer (Q Exactive, Thermo Scientific, Boston, MA) as previously described ${ }^{79}$. Briefly, peptides were separated on a reversed-phase tip column $(150 \mu \mathrm{m}$ i.d. $\times 70 \mathrm{~mm}$, Mightysil-C18, $3-\mu \mathrm{m}$ particle) using a $0-35 \%$ linear gradient of acetonitrile in $0.1 \%(\mathrm{v} / \mathrm{v})$ formic acid for 35 or $70 \mathrm{~min}$ at a flow rate of $100 \mathrm{~nL} / \mathrm{min}$. Full MS scans were acquired with a resolution of 30,000 at a mass-to-charge ratio of 400 . The ten most intense ions were fragmented in the data-dependent mode by collision-induced dissociation with normalized collision energy of 35 , activation q of 0.25 , and activation time of $10 \mathrm{~ms}$ and one microscan. The MS/MS data were converted to the mascot generic format with the Proteome Discoverer software (Thermo Scientific, ver. 1.1). The files were processed with the MASCOT algorithm (version 2.2.1., Matrix Science Ltd., London, United Kingdom) to assign peptides using the Swiss-Prot sequence database (release 2012.11, human) under the search parameters described in $\operatorname{Ref}^{79}$. Peptides were identified based on the MASCOT definitions. For the search parameters, we set the variable modifications for acetylation (protein $\mathrm{N}$ terminus) and oxidation (Met). The 
maximum missed cleavage was set at 1 with a peptide mass tolerance of $+/-15 \mathrm{ppm}$. Peptide charges from +2 to +4 states and MS/MS tolerances of $+/-0.8$ Da were allowed. All results of peptide searches were extracted from the Mascot DAT files using the STEM software ${ }^{80}$.

\section{Cholesterol depletion}

Lipoprotein-deficient serum (LPDS) was prepared as described previously ${ }^{81,82}$. In brief, FCS was adjusted to a density of $1.25 \mathrm{~g} / \mathrm{mL}$ with solid $\mathrm{KBr}$ and then centrifuged for 16 $\mathrm{h}$ at $10^{\circ} \mathrm{C}$ at $50,000 \mathrm{rpm}$ in a Beckman VTi 50 rotor. The upper yellow-orange layer containing lipoproteins and the salt pellet were removed, and the remaining fraction was dialyzed extensively at $4^{\circ} \mathrm{C}$ against $150 \mathrm{mM} \mathrm{NaCl}$ for $48 \mathrm{~h}$. HeLa cells were incubated with DMEM containing 5\% LPDS and 1\% 2-hydroxypropyl- $\beta$-cyclodextrin for $3 \mathrm{~h}$.

\section{Quantitative real-time PCR}

RNA was prepared using an RNeasy Mini kit (QIAGEN) and cDNA was synthesized with SuperScript III reverse transcriptase (Invitrogen) primed by oligo(dT)15. Quantitative real-time PCR was performed with a Rotor-Gene Q RT-PCR machine (QIAGEN) using a KAPA SYBR FAST qPCR kit (Kapa Biosystems), according to the manufacturer's protocol. The primers used were as follows: SCAP, forward primer 5'TATCTCGGGCCTTCTACAACC-3' and reverse primer 5'GGGGCGAGTAATCCTTCACA-3'; HMGR, forward primer 5'TGACCTTTCCAGAGCAAGC-3' and reverse primer 5'CCAACTCCAATCACAAGACATTC-3'; LDLR, forward primer 5'GTGTCACAGCGGCGAATG-3' and reverse primer 5'CGCACTCTTTGATGGGTTCA-3'; HPRT1, forward primer 5'TTCCAGACAAGTTTGTTGTAGGAT-3' and reverse primer 5'GCAGATGGCCACAGAACTAG-3'. Values were normalized as the HPRT1 expression level. 


\section{Total cholesterol measurement}

HeLa cells were lysed in water for $30 \mathrm{~min}$ at $37^{\circ} \mathrm{C}$ and then subjected to total cholesterol measurement with an Amplex Red cholesterol assay kit (Molecular probes), according to the manufacturer's protocol. Values were normalized to protein content determined as using a Pierce BCA protein assay kit (Thermo Fisher Scientific).

\section{PAUF secretion assay}

HeLa cells were transfected with control siRNA or siRNA oligos targeting SCAP. At $48 \mathrm{~h}$ after siRNA transfection, the cells were transfected with a plasmid for PAUFMycHis, $20 \mathrm{~h}$ later the medium was replaced with Opti-MEM, and then the cells were incubated at $37^{\circ} \mathrm{C}$ for $6 \mathrm{~h}$. After collecting the medium, the cells were lysed with $0.5 \%$ sodium dodecyl sulfate (SDS) and 0.025 units $\mu \mathrm{L}$ benzonase nuclease (Sigma-Aldrich) in PBS. The medium and cell lysates were analyzed by Western blotting with an antiPenta-His antibody.

\section{GPI transport assay}

HeLa cells were transfected with control siRNA or siRNA oligos targeting SCAP, VAP-A/VAP-B, OSBP or CERT/OSBP. At $48 \mathrm{~h}$ after siRNA transfection, the cells were transfected with a plasmid for mKate2-FM4-GPI, $20 \mathrm{~h}$ later the medium was replaced with DMEM containing 10\% FCS, $1 \mu \mathrm{M}$ D/D solubilizer, and $20 \mu \mathrm{g} / \mathrm{mL}$ cycloheximide, and then cells were incubated at $37^{\circ} \mathrm{C}$ for the indicated times. The cells were then fixed with 4\% PFA and analyzed by fluorescence microscopy as described above.

\section{Inducible CARTS formation assay}

HeLa cells were transfected with control siRNA or siRNA oligos targeting SCAP, SREBP1, or SREBP2. At $48 \mathrm{~h}$ after siRNA transfection, the cells were transfected with a plasmid for mKate2-FM4-PAUF, $20 \mathrm{~h}$ later the medium was replaced with DMEM containing 10\% FCS, $20 \mathrm{mM}$ HEPES-KOH, pH 7.4, $1 \mu \mathrm{M}$ D/D solubilizer, and 20 $\mu \mathrm{g} / \mathrm{mL}$ cycloheximide, and then the cells were incubated in a water bath at $20^{\circ} \mathrm{C}$ for 45 
$\min$. The cells were then incubated in a water bath at $37^{\circ} \mathrm{C}$ for the indicated times, followed by fixation with 4\% PFA. The samples were analyzed by fluorescence microscopy as described above. Quantification of CARTS was performed with ImageJ software (National Institutes of Health). The fluorescence signal of mKate2-FM4PAUF-containing puncta was distinguished from the background by setting a threshold and analyzed at a set size within $0.05-2.00 \mu \mathrm{m}^{2}$. For live-cell imaging, HeLa cells were transfected with a plasmid for mKate2-FM4-PAUF, $20 \mathrm{~h}$ later the medium was replaced with Opti-MEM containing $1 \mu \mathrm{M}$ D/D solubilizer and $20 \mu \mathrm{g} / \mathrm{mL}$ cycloheximide, and then the cells were incubated in a water bath at $20^{\circ} \mathrm{C}$ for $45 \mathrm{~min}$. The cells were then incubated at $37^{\circ} \mathrm{C}$ and $5 \% \mathrm{CO}_{2}$ to monitor CARTS biogenesis. Images were acquired continuously with a time interval between frames of $30 \mathrm{sec}$ for $\sim 75 \mathrm{~min}$ by use of an Olympus Fluoview FV1200 confocal microscope with a UPLSAPO 60x O NA 1.35 objective and FV10-ASW software. The images were processed with Image J software. 


\section{Acknowledgements}

We thank Peter Espenshade, Peter Mayinger, Hiroyuki Arai, Kentaro Hanada, Nobuhiro Nakamura, Sang Seok Koh, David M. Sabatini, Cristopher G. Burd, and Vivek Malhotra for providing materials. We appreciate the technical assistance of Nanako Oyama, So Yoshida, Yuika Komatsuda, Yuiko Kawai, Natsumi Hoshino, Tomoya Iizuka, Sho Furuichi, and Katsunori Iwasa. We are grateful to Josse van Galen and Vivek Malhotra for comments on the manuscript. This work was supported in part by Grants-in-Aid for Scientific Research [grant numbers 15K18507 and 17K07348 to Y.W., and 18H02439 to M.T.] from the Ministry of Education, Culture, Sports, Science, and Technology of Japan, the Naito Foundation [to Y.W.], and the Ono Medical Research Foundation [to Y.W.]. F.C. acknowledges financial support from the Spanish Ministry of Economy and Competitiveness ("Severo Ochoa" program for Centres of Excellence in R\&D (SEV-2015-0522), FIS2015 - 63550 - R, FIS2017 - 89560 - R, BFU2015 - 73288 - JIN, AEI/FEDER/UE, and RYC-2017-22227), Fundació Privada Cellex, and from the Generalitat de Catalunya through the CERCA program.

\section{Author contributions}

Y.W. and M.Tagaya conceived and designed the experiments. Y.W., K.H., T.N., C.W., F.C., and H.K. performed the experiments. M.Taoka performed LC-MS/MS analysis and analyzed the data. T.U., H.I., K.A., and M.Tagaya provided samples. Y.W., F.C., and M.Tagaya wrote the manuscript.

\section{Competing interests}

The authors declare no competing interests. 


\section{References}

1. Simons, K. \& Ikonen, E. Functional rafts in cell membranes. 387, 4 (1997).

2. Keller, P. \& Simons, K. Cholesterol Is Required for Surface Transport of Influenza Virus Hemagglutinin. J. Cell Biol. 140, 1357-1367 (1998).

3. Wang, Y., Thiele1, C. \& Huttner, W. B. Cholesterol is Required for the Formation of Regulated and Constitutive Secretory Vesicles from the trans-Golgi Network. Traffic 1, 952-962 (2000).

4. Ikonen, E. Roles of lipid rafts in membrane transport. Curr. Opin. Cell Biol. 13, 470-477 (2001).

5. Klemm, R. W. et al. Segregation of sphingolipids and sterols during formation of secretory vesicles at the trans-Golgi network. J. Cell Biol. 185, 601-612 (2009).

6. Lingwood, D. \& Simons, K. Lipid Rafts As a Membrane-Organizing Principle. Science 327, 46-50 (2010).

7. Simons, K. \& Sampaio, J. L. Membrane organization and lipid rafts. Cold Spring Harb. Perspect. Biol. 3, a004697 (2011).

8. Jacobson, K., Liu, P. \& Lagerholm, B. C. The Lateral Organization and Mobility of Plasma Membrane Components. Cell 177, 806-819 (2019).

9. Duran, J. M. et al. Sphingomyelin organization is required for vesicle biogenesis at the Golgi complex: Lipid organization controls vesicle biogenesis. EMBO J. 31, 4535-4546 (2012).

10. van Galen, J. et al. Sphingomyelin homeostasis is required to form functional enzymatic domains at the trans-Golgi network. J. Cell Biol. 206, 609-618 (2014).

11. Deng, Y., Rivera-Molina, F. E., Toomre, D. K. \& Burd, C. G. Sphingomyelin is sorted at the trans Golgi network into a distinct class of secretory vesicle. Proc. Natl. Acad. Sci. 113, 6677-6682 (2016).

12. Campelo, F. et al. Sphingomyelin metabolism controls the shape and function of the Golgi cisternae. eLife 6, (2017).

13. Capasso, S. et al. Sphingolipid metabolic flow controls phosphoinositide turnover at the trans -Golgi network. EMBO J. 36, 1736-1754 (2017).

14. Deng, Y. et al. Activity of the SPCA1 Calcium Pump Couples Sphingomyelin Synthesis to Sorting of Secretory Proteins in the Trans-Golgi Network. Dev. Cell 47, 464-478.e8 (2018). 
15. Hua, X., Nohturfft, A., Goldstein, J. L. \& Brown, M. S. Sterol Resistance in CHO Cells Traced to Point Mutation in SREBP Cleavage-Activating Protein. Cell 87, 415-426 (1996).

16. Brown, M. S. \& Goldstein, J. L. The SREBP Pathway: Regulation of Cholesterol Metabolism by Proteolysis of a Membrane-Bound Transcription Factor. Cell 89, 331340 (1997).

17. Goldstein, J. L., DeBose-Boyd, R. A. \& Brown, M. S. Protein Sensors for Membrane Sterols. Cell 124, 35-46 (2006).

18. Radhakrishnan, A., Goldstein, J. L., McDonald, J. G. \& Brown, M. S. Switchlike Control of SREBP-2 Transport Triggered by Small Changes in ER Cholesterol: A Delicate Balance. Cell Metab. 8, 512-521 (2008).

19. Brown, M. S. \& Goldstein, J. L. Cholesterol feedback: from Schoenheimer's bottle to Scap's MELADL. J. Lipid Res. 50, S15-S27 (2009).

20. Brown, M. S., Radhakrishnan, A. \& Goldstein, J. L. Retrospective on Cholesterol Homeostasis: The Central Role of Scap. Annu. Rev. Biochem. 87, 783807 (2018).

21. Ikonen, E. Mechanisms of cellular cholesterol compartmentalization: recent insights. Curr. Opin. Cell Biol. 53, 77-83 (2018).

22. Mesmin, B. et al. A Four-Step Cycle Driven by PI(4)P Hydrolysis Directs Sterol/PI(4)P Exchange by the ER-Golgi Tether OSBP. Cell 155, 830-843 (2013).

23. Antonny, B., Bigay, J. \& Mesmin, B. The Oxysterol-Binding Protein Cycle: Burning Off PI(4)P to Transport Cholesterol. Annu. Rev. Biochem. 87, 809-837 (2018).

24. Hanada, K. et al. Molecular machinery for non-vesicular trafficking of ceramide. Nature 426, 803-809 (2003).

25. Kawano, M., Kumagai, K., Nishijima, M. \& Hanada, K. Efficient Trafficking of Ceramide from the Endoplasmic Reticulum to the Golgi Apparatus Requires a VAMP-associated Protein-interacting FFAT Motif of CERT. J. Biol. Chem. 281, 30279-30288 (2006).

26. Hanada, K., Kumagai, K., Tomishige, N. \& Yamaji, T. CERT-mediated trafficking of ceramide. Biochim. Biophys. Acta BBA - Mol. Cell Biol. Lipids 1791, 684-691 (2009).

27. De Matteis, M. A., Di Campli, A. \& Godi, A. The role of the phosphoinositides at the Golgi complex. Biochim. Biophys. Acta 1744, 396-405 (2005). 
28. Kumagai, K. \& Hanada, K. Structure, functions and regulation of CERT, a lipidtransfer protein for the delivery of ceramide at the ER-Golgi membrane contact sites. FEBS Lett. 0,

29. Fugmann, T. et al. Regulation of secretory transport by protein kinase Dmediated phosphorylation of the ceramide transfer protein. J. Cell Biol. 178, 15-22 (2007).

30. Nhek, S. et al. Regulation of Oxysterol-binding Protein Golgi Localization through Protein Kinase D-mediated Phosphorylation. Mol. Biol. Cell 21, 2327-2337 (2010).

31. Baron, C. L. \& Malhotra, V. Role of Diacylglycerol in PKD Recruitment to the TGN and Protein Transport to the Plasma Membrane. Science 295, 325-328 (2002).

32. Malhotra, V. \& Campelo, F. PKD Regulates Membrane Fission to Generate TGN to Cell Surface Transport Carriers. Cold Spring Harb. Perspect. Biol. 3, a005280-a005280 (2011).

33. Pagliuso, A. et al. Golgi membrane fission requires the CtBP1-S/BARS-induced activation of lysophosphatidic acid acyltransferase $\delta$. Nat. Commun. 7, 12148 (2016).

34. Wakana, Y. et al. CARTS biogenesis requires VAP-lipid transfer protein complexes functioning at the endoplasmic reticulum-Golgi interface. Mol. Biol. Cell 26, 4686-4699 (2015).

35. Wakana, Y. et al. A new class of carriers that transport selective cargo from the trans Golgi network to the cell surface. EMBO J. 31, 3976-3990 (2012).

36. Wakana, Y. et al. Kinesin-5/Eg5 is important for transport of CARTS from the trans-Golgi network to the cell surface. J. Cell Biol. 202, 241-250 (2013).

37. Blagoveshchenskaya, A. et al. Integration of Golgi trafficking and growth factor signaling by the lipid phosphatase SAC1. J. Cell Biol. 180, 803-812 (2008).

38. Gomez-Navarro, N. \& Miller, E. Protein sorting at the ER-Golgi interface. $J$. Cell Biol. 215, 769-778 (2016).

39. Wakana, Y. et al. Bap31 Is an Itinerant Protein That Moves between the Peripheral Endoplasmic Reticulum (ER) and a Juxtanuclear Compartment Related to ER-associated Degradation. Mol. Biol. Cell 19, 1825-1836 (2008).

40. Lavieu, G. et al. Induction of cortical endoplasmic reticulum by dimerization of a coatomer-binding peptide anchored to endoplasmic reticulum membranes. Proc.

Natl. Acad. Sci. 107, 6876-6881 (2010). 
41. Ridgway, N. D., Dawson, P. A., Ho, Y. K., Brown, M. S. \& Goldstein, J. L. Translocation of oxysterol binding protein to Golgi apparatus triggered by ligand binding. J. Cell Biol. 116, 307-319 (1992).

42. Suchanek, M. et al. The mammalian oxysterol-binding protein-related proteins (ORPs) bind 25-hydroxycholesterol in an evolutionarily conserved pocket. Biochem. J. 405, 473-480 (2007).

43. Yang, T. et al. Crucial Step in Cholesterol Homeostasis: Sterols Promote Binding of SCAP to INSIG-1, a Membrane Protein that Facilitates Retention of SREBPs in ER. Cell 110, 489-500 (2002).

44. Wyles, J. P., McMaster, C. R. \& Ridgway, N. D. Vesicle-associated Membrane Protein-associated Protein-A (VAP-A) Interacts with the Oxysterol-binding Protein to Modify Export from the Endoplasmic Reticulum. J. Biol. Chem. 277, 29908-29918 (2002).

45. Loewen, C. J. R. A conserved ER targeting motif in three families of lipid binding proteins and in Opilp binds VAP. EMBO J. 22, 2025-2035 (2003).

46. Nohturfft, A., Brown, M. S. \& Goldstein, J. L. Topology of SREBP Cleavageactivating Protein, a Polytopic Membrane Protein with a Sterol-sensing Domain. $J$. Biol. Chem. 273, 17243-17250 (1998).

47. Sun, L.-P., Li, L., Goldstein, J. L. \& Brown, M. S. Insig Required for Sterolmediated Inhibition of Scap/SREBP Binding to COPII Proteins in Vitro. J. Biol. Chem. 280, 26483-26490 (2005).

48. Kentala, H., Pfisterer, S. G., Olkkonen, V. M. \& Weber-Boyvat, M. Sterol liganding of OSBP-related proteins (ORPs) regulates the subcellular distribution of ORP-VAPA complexes and their impacts on organelle structure. Steroids 99, 248258 (2015).

49. Weber-Boyvat, M., Kentala, H., Peränen, J. \& Olkkonen, V. M. Liganddependent localization and function of ORP-VAP complexes at membrane contact sites. Cell. Mol. Life Sci. 72, 1967-1987 (2015).

50. Nohturfft, A., Yabe, D., Goldstein, J. L., Brown, M. S. \& Espenshade, P. J. Regulated Step in Cholesterol Feedback Localized to Budding of SCAP from ER Membranes. Cell 102, 315-323 (2000).

51. Horton, J. D. et al. Combined analysis of oligonucleotide microarray data from transgenic and knockout mice identifies direct SREBP target genes. Proc. Natl. Acad. Sci. 100, 12027-12032 (2003). 
52. Dong, R. et al. Endosome-ER Contacts Control Actin Nucleation and Retromer Function through VAP-Dependent Regulation of PI4P. Cell 166, 408-423 (2016).

53. Rivera, V. M. et al. Regulation of Protein Secretion Through Controlled Aggregation in the Endoplasmic Reticulum. Science 287, 826-830 (2000).

54. Mayor, S. \& Riezman, H. Sorting GPI-anchored proteins. Nat. Rev. Mol. Cell Biol. 5, 110 (2004).

55. Brown, D. A. \& Rose, J. K. Sorting of GPI-anchored proteins to glycolipidenriched membrane subdomains during transport to the apical cell surface. Cell $\mathbf{6 8}$, 533-544 (1992).

56. Radhakrishnan, A., Sun, L.-P., Kwon, H. J., Brown, M. S. \& Goldstein, J. L. Direct Binding of Cholesterol to the Purified Membrane Region of SCAP: Mechanism for a Sterol-Sensing Domain. Mol. Cell 15, 259-268 (2004).

57. Motamed, M. et al. Identification of luminal loop 1 of scap as the sterol sensor that maintains cholesterol homeostasis. J. Biol. Chem. jbc.M111.238311 (2011) doi:10.1074/jbc.M111.238311.

58. Nohturfft, A., Brown, M. S. \& Goldstein, J. L. Sterols regulate processing of carbohydrate chains of wild-type SREBP cleavage-activating protein (SCAP), but not sterol-resistant mutants Y298C or D443N. Proc. Natl. Acad. Sci. 95, 12848-12853 (1998).

59. Brown, A. J., Sun, L., Feramisco, J. D., Brown, M. S. \& Goldstein, J. L. Cholesterol Addition to ER Membranes Alters Conformation of SCAP, the SREBP Escort Protein that Regulates Cholesterol Metabolism. Mol. Cell 10, 237-245 (2002). 60. Yabe, D., Brown, M. S. \& Goldstein, J. L. Insig-2, a second endoplasmic reticulum protein that binds SCAP and blocks export of sterol regulatory elementbinding proteins. Proc. Natl. Acad. Sci. 99, 12753-12758 (2002).

61. Adams, C. M., Goldstein, J. L. \& Brown, M. S. Cholesterol-induced conformational change in SCAP enhanced by Insig proteins and mimicked by cationic amphiphiles. Proc. Natl. Acad. Sci. 100, 10647-10652 (2003).

62. Matsuda, M. et al. SREBP cleavage-activating protein (SCAP) is required for increased lipid synthesis in liver induced by cholesterol deprivation and insulin elevation. Genes Dev. 15, 1206-1216 (2001).

63. Rawson, R. B., DeBose-Boyd, R., Goldstein, J. L. \& Brown, M. S. Failure to Cleave Sterol Regulatory Element-binding Proteins (SREBPs) Causes Cholesterol 
Auxotrophy in Chinese Hamster Ovary Cells with Genetic Absence of SREBP

Cleavage-activating Protein. J. Biol. Chem. 274, 28549-28556 (1999).

64. Moon, Y.-A. et al. The Scap/SREBP Pathway Is Essential for Developing

Diabetic Fatty Liver and Carbohydrate-Induced Hypertriglyceridemia in Animals.

Cell Metab. 15, 240-246 (2012).

65. Friedman, J. R. et al. ER Tubules Mark Sites of Mitochondrial Division. Science 334, 358-362 (2011).

66. Rowland, A. A., Chitwood, P. J., Phillips, M. J. \& Voeltz, G. K. ER Contact Sites Define the Position and Timing of Endosome Fission. Cell 159, 1027-1041 (2014).

67. Pakdel, M. \& von Blume, J. Exploring new routes for secretory protein export from the trans-Golgi network. Mol. Biol. Cell 29, 235-240 (2018).

68. Adams, C. M. et al. Cholesterol and 25-Hydroxycholesterol Inhibit Activation of SREBPs by Different Mechanisms, Both Involving SCAP and Insigs. J. Biol. Chem. 279, 52772-52780 (2004).

69. Radhakrishnan, A., Ikeda, Y., Kwon, H. J., Brown, M. S. \& Goldstein, J. L. Sterol-regulated transport of SREBPs from endoplasmic reticulum to Golgi:

Oxysterols block transport by binding to Insig. Proc. Natl. Acad. Sci. 104, 6511-6518 (2007).

70. Gong, Y., Lee, J. N., Brown, M. S., Goldstein, J. L. \& Ye, J. Juxtamembranous aspartic acid in Insig-1 and Insig-2 is required for cholesterol homeostasis. Proc. Natl. Acad. Sci. 103, 6154-6159 (2006).

71. Nishimura, T. et al. Oxysterol-binding protein (OSBP) is required for the perinuclear localization of intra-Golgi v-SNAREs. Mol. Biol. Cell 24, 3534-3544 (2013).

72. Kumagai, K., Kawano, M., Shinkai-Ouchi, F., Nishijima, M. \& Hanada, K. Interorganelle Trafficking of Ceramide Is Regulated by Phosphorylation-dependent Cooperativity between the PH and START Domains of CERT. J. Biol. Chem. 282, 17758-17766 (2007).

73. Peterson, T. R. et al. mTOR Complex 1 Regulates Lipin 1 Localization to Control the SREBP Pathway. Cell 146, 408-420 (2011).

74. Wilhelm, L. P. et al. STARD3 mediates endoplasmic reticulum-to-endosome cholesterol transport at membrane contact sites. EMBO J. 36, 1412-1433 (2017). 
75. Hammond, G. R. V., Schiavo, G. \& Irvine, R. F. Immunocytochemical techniques reveal multiple, distinct cellular pools of PtdIns4 $P$ and PtdIns(4,5) $P_{2}$. Biochem. J. 422, 23-35 (2009).

76. Schindelin, J. et al. Fiji: an open-source platform for biological-image analysis. Nat. Methods 9, 676-682 (2012).

77. Venditti, R. et al. The activity of Sac1 across ER-TGN contact sites requires the four-phosphate-adaptor-protein-1. J. Cell Biol. jcb.201812021 (2019) doi:10.1083/jcb.201812021.

78. Taoka, M., Wakamiya, A., Nakayama, H. \& Isobe, T. Protein profiling of rat cerebella during development. ELECTROPHORESIS 21, 1872-1879 (2000).

79. Taoka, M. et al. Global PROTOMAP profiling to search for biomarkers of earlyrecurrent hepatocellular carcinoma. J. Proteome Res. 13, 4847-4858 (2014).

80. Shinkawa, T. et al. STEM: A Software Tool for Large-Scale Proteomic Data Analyses. J. Proteome Res. 4, 1826-1831 (2005).

81. Goldstein, J. L., Basu, S. K. \& Brown, M. S. [19] Receptor-mediated endocytosis of low-density lipoprotein in cultured cells. in Methods in Enzymology vol. 98 241260 (Elsevier, 1983).

82. Poumay, Y. \& Ronveaux-Dupal, M. F. Rapid preparative isolation of concentrated low density lipoproteins and of lipoprotein-deficient serum using vertical rotor gradient ultracentrifugation. J. Lipid Res. 26, 1476-1480 (1985). 


\section{Figure legends}

Fig. 1 | Identification of SCAP as a novel component of Sac1-positive ER-Golgi contact sites. a, Juxtanuclear localization of the GFP-Sac1 WT and K2A mutant in HeLa cells. b, Colocalization of GFP-Sac1 WT or K2A with VAP-A and their proximity localization with TGN46. HeLa cells expressing GFP-Sac1 WT or K2A were treated with $2 \mu \mathrm{g} / \mathrm{mL} 25-\mathrm{HC}$ for $1 \mathrm{~h}$. Images were subjected to deconvolution processing as described in Materials and methods. The graphs on the right show the fluorescence intensity of GFP-Sac1 WT or K2A (green), and VAP-A or TGN46 (magenta) along the respective white lines shown in the merged images. $\mathrm{N}$, nucleus. c, iFRAP in HeLa cells expressing the GFP-Sac1 WT, K2A mutant, or Nacetylglucosaminyl transferase I (NA)-GFP. The areas delimited by a red line were bleached as described in Materials and methods. The graph below shows quantification of the fluorescence intensity of the indicated proteins in the non-bleached, juxtanuclear region. Data are means \pm s.e.m. $(\mathrm{n}=4$ cells per condition, $* * * * P<0.0001$, one-way ANOVA multiple comparison test). d, Silver staining of immunoprecipitated proteins with the FLAG-Sac1 WT, K2A, or FLAG in HEK 293T cells. Asterisks denote protein bands containing COPI components. SCAP was identified in the protein band boxed with a red line. e, Peptides of VAP-A, VAP-B, OSBP, and SCAP, which were identified by mass spectrometric analysis of FLAG-Sac1 K2A immunoprecipitates (lane 2 in panel d). Scale bars, $10 \mu \mathrm{m}$.

\section{Fig. 2 | ER-localized SCAP interacts through Sac1 with the VAP-A/OSBP complex} at ER-Golgi contact sites. a, Interactions of FLAG-SCAP with Myc-OSBP, Sac1, and VAP-A in HEK 293T cells. Cell lysates were subjected to immunoprecipitation with an anti-FLAG M2 affinity gel, and the cell lysates (Input) and immunoprecipitates (IP) were immunoblotted (IB) with the indicated antibodies. Asterisks denote degraded Myc-OSBP fragments. b, Interactions of FLAG-SCAP with Myc-OSBP, Sac1, and VAP-A, but not with HA-CERT or RTN-4B. c, Interactions of FLAG-SCAP C-term with Myc-OSBP, Sac1, and VAP-A. d, Interactions of the FLAG-SCAP D451A/L452A mutant with Myc-OSBP, Sac1, and VAP-A. e-j, BiFC visualization of SCAP/Sac1 or 
SCAP/VAP-A interactions at ER-Golgi contact sites in HeLa cells coexpressing MycOSBP and Venus N-terminal fragment (Vn)-fused SCAP in combination with Venus C-terminal fragment (Vc)-fused $\operatorname{Sac} 1(\mathbf{e}, \mathbf{g}, \mathbf{i})$ or VAP-A $(\mathbf{f}, \mathbf{h}, \mathbf{j})$. The cells were treated without [Control (Cont)] or with $2 \mu \mathrm{g} / \mathrm{mL} 25-\mathrm{HC}$ for $2.5 \mathrm{~h}$, or were cholesterol depleted (Chol Depl) for $3 \mathrm{~h}$. The expression of Vn-SCAP together with Vc-Sac1 (e) or VcVAP-A (f) was visualized with an anti-GFP antibody. TGN46 was visualized with antiTGN46 antibody $(\mathbf{i}, \mathbf{j})$. N, nucleus. The box-and-whisker plots show quantification of perinuclear BiFC signal $(\mathbf{g}, \mathbf{h})$. Boxes delimit the first and third quartiles and the central line is the median, whereas the cross represents the mean value. The whiskers represent the minimum and maximum values after outlier removal (Tukey whiskers) (VnSCAP/Vc-Sac1: control, $\mathrm{n}=86$ cells; $25-\mathrm{HC}, \mathrm{n}=70$ cells; cholesterol depleted, $\mathrm{n}=$ 101 cells, Vn-SCAP/Vc-VAP-A: control, $\mathrm{n}=93$ cells; $25-\mathrm{HC}, \mathrm{n}=92$ cells; cholesterol depleted, $\mathrm{n}=101$ cells, $* \mathrm{P}<0.05, * * * * P<0.0001$, NS, not significant, Kruskal-Wallis multiple sample non-parametric test). Scale bars, $10 \mu \mathrm{m}$.

\section{Fig. 3 | SCAP knockdown does not disrupt cholesterol metabolism under} cholesterol-fed conditions. a, siRNA-mediated knockdown of SCAP in HeLa cells, monitored by Western blotting. The graph shows determination of the expression levels of SCAP at $72 \mathrm{~h}$ after siRNA transfection. Data are means \pm s.e.m. $(\mathrm{n}=3$ independent experiments, ${ }^{* * *} P<0.001$, unpaired two-tailed Student's $t$-test). Asterisks on the Western blot denote nonspecific bands. b, Determination of the mRNA levels of the indicated genes in control (Cont) and SCAP knockdown cells by quantitative real-time PCR. Data are means \pm s.e.m. $(\mathrm{n}=4$ independent experiments, $* * P<0.01, * * * P<$ 0.005 , NS, not significant, unpaired two-tailed Student's $t$-test). c, Total cholesterol (Chol) levels in control and SCAP knockdown cells. Data are means \pm s.e.m. ( $n=6$ independent experiments, NS, not significant, unpaired two-tailed Student's $t$-test). d,e, shRNA-mediated knockdown of SCAP in HeLa cells, monitored by Western blotting. The graph (e) shows determination of the expression levels of SCAP, HMGR, and LDLR in parental HeLa (control) and shSCAP HeLa cells, as measured by Western blotting. Data are mean \pm s.e.m. $(\mathrm{n}=4$ independent experiments, $* P<0.05$, $* * * * P<$ 
0.001, NS, not significant, unpaired two-tailed Student's $t$-test). Asterisks on the Western blot denote nonspecific bands. f, Total cholesterol levels in parental HeLa and shSCAP HeLa cells. Data are means \pm s.e.m. $(n=3$ independent experiments, NS, not significant, unpaired two-tailed Student's $t$-test).

Fig. 4 | SCAP is important for PI4P turnover and VAP-A/OSBP complex distribution at ER-Golgi contact sites. a, Filipin staining in parental HeLa and shSCAP HeLa cells. High magnifications of the boxed areas are shown in the right panels. Scale bars, $10 \mu \mathrm{m}$. b,c, PI4P staining in parental HeLa and shSCAP HeLa cells with (top two rows in $\mathbf{c}$ ) or without (b and bottom two rows in $\mathbf{c}$ ) sialyltransferase (ST)mRFP expression. N, nucleus. Scale bars, $10 \mu \mathrm{m}$. The graph shows determination of the Golgi PI4P levels in parental HeLa (control) and shSCAP HeLa cells. Data are means \pm s.e.m. $(\mathrm{n}=139$ cells per conditions, $* * * * P<0.0001$, unpaired two-tailed Student's $t$-test). d, BiFC visualization of OSBP/VAP-A interactions in control (Cont) and SCAP knockdown cells. HeLa cells stably coexpressing Vn-OSBP and Vc-VAPA were transfected with siRNA. After $72 \mathrm{~h}$, the cells were treated with or without 2 $\mu \mathrm{g} / \mathrm{mL} 25-\mathrm{HC}$ for $2.5 \mathrm{~h}$. High magnifications of the boxed areas are shown in the insets where brightness/contrast-enhancement was applied. Scale bars, $10 \mu \mathrm{m}$ (large panels), $5 \mu \mathrm{m}$ (insets). The graph shows the percentage of cells with the peripheral BiFC signal of Vn-OSBP/Vc-VAP-A. Data are means \pm s.e.m. $(\mathrm{n}=3$ independent experiments, 100 131 cells per condition, ${ }^{* * *} P<0.005$, unpaired two-tailed Student's $t$-test). e, Close apposition of CD63- but not of TGN46-positive membranes to the peripheral BiFC signal of Vn-OSBP/Vc-VAP-A in SCAP knockdown cells treated with $2 \mu \mathrm{g} / \mathrm{mL} 25$ HC for $2.5 \mathrm{~h}$. High magnifications of the boxed areas are shown in the insets. Scale bars, $10 \mu \mathrm{m}$ (large panels), $5 \mu \mathrm{m}$ (insets).

Fig. 5 | SCAP is required for the biogenesis of CARTS at the TGN. a, Close proximity of CARTS formation sites to VAP-A/OSBP-mediated ER-Golgi contact sites. HeLa cells stably coexpressing Vn-OSBP and Vc-VAP-A were transfected with a plasmid for PAUF-MycHis. After $20 \mathrm{~h}$, the cells were treated with $2 \mu \mathrm{g} / \mathrm{mL} 25-\mathrm{HC}$ for 
2.5 h. Images were subjected to deconvolution processing as described in Materials and methods. N, nucleus. High magnifications of the boxed areas are shown in the bottom row. Arrowheads indicate putative nascent CARTS located in the close vicinity of the BiFC signal of Vn-OSBP/Vc-VAP-A. Scale bars, $5 \mu \mathrm{m}$ (upper row), $2.5 \mu \mathrm{m}$ (bottom row). b, PAUF-MycHis secretion in control (Cont) and SCAP knockdown cells, monitored by Western blotting. The graph shows quantification of secreted PAUFMycHis relative to the total cellular level and normalized as the values in control cells. Data are means \pm s.e.m. $\left(\mathrm{n}=3\right.$ independent experiments, ${ }^{*} P<0.01$, unpaired twotailed Student's $t$-test). c, Biogenesis of mKate2-FM4-PAUF-containing CARTS in control and SCAP knockdown cells. The cells were incubated at $20^{\circ} \mathrm{C}$ with the $\mathrm{D} / \mathrm{D}$ solubilizer and cycloheximide (CHX) for $45 \mathrm{~min}$, followed by incubation at $37^{\circ} \mathrm{C}$ for 5 or 15 min. The graph shows the number of mKate2-FM4-PAUF-containing CARTS in control and SCAP knockdown cells at 15 min after the temperature shift to $37^{\circ} \mathrm{C}$. Data are means \pm s.e.m. (control siRNA: $\mathrm{n}=20$ cells; SCAP siRNA: $\mathrm{n}=29$ cells, $* * * * P<$ 0.0001 , unpaired two-tailed Student's $t$-test). Scale bar, $10 \mu \mathrm{m}$.

Fig. 6 | SCAP is required for GPI-anchored protein transport from the TGN to the PM. a, mKate2-FM4-GPI transport from the ER to the PM via the Golgi complex in control, SCAP, and VAP-A/B knockdown cells. The cells were incubated at $37^{\circ} \mathrm{C}$ with the D/D solubilizer and cycloheximide, and fixed at the indicated times. The graph shows the percentages of cells with mKate2-FM4-GPI at the ER, ER/Golgi, Golgi/ PM, or PM at the indicated times. The data shown are for a single representative experiment out of three performed (control siRNA: $30 \mathrm{~min}, \mathrm{n}=235$ cells; $60 \mathrm{~min}, \mathrm{n}=239$ cells; 90 min, $\mathrm{n}=252$ cells; SCAP siRNA: $30 \mathrm{~min}, \mathrm{n}=248$ cells; $60 \mathrm{~min}, \mathrm{n}=246$ cells; $90 \mathrm{~min}$, $\mathrm{n}=241$ cells; VAP-A/B siRNA: 30 min, $\mathrm{n}=230$ cells; $60 \mathrm{~min}, \mathrm{n}=238$ cells; $90 \mathrm{~min}$, $\mathrm{n}=240$ cells). b, mKate2-FM4-GPI has accumulated at the TGN at $90 \mathrm{~min}$ after the transport induction in SCAP knockdown cells. N, nucleus. c, Colocalization of PAUFMycHis and mKate2-FM4-GPI in CARTS at $30 \mathrm{~min}$ after transport induction. d, Colocalization of GST-PKD2-kinase dead (KD) and mKate2-FM4-GPI in tubules attached to the TGN at 90 min after transport induction. In $\mathbf{c}$ and $\mathbf{d}$, high magnifications 
of the boxed areas are shown in the right column where brightness/contrastenhancement was applied to the mKate2-FM4-GPI channel. Arrowheads in $\mathbf{d}$ indicate a GST-PKD2-KD-induced tubule containing mKate2-FM4-GPI. e, Colocalization of EQ-SM (tagged with oxGFP) and PAUF-MycHis. High magnifications of the boxed areas are shown in the insets. The box-and-whisker plots show quantification of EQSM-positive puncta containing PAUF-MycHis (green) and PAUF-MycHis-positive puncta (CARTS) containing EQ-SM (magenta). Boxes delimit the first and third quartiles and the central line is the median. The whiskers represent the minimum and maximum values after outlier removal (Tukey whiskers) (EQ-SM-positive: $n=4487$ puncta, PAUF-positive: $\mathrm{n}=1650$ puncta in 12 cells, $* * * * P<0.0001$, paired two-tailed Student's $t$-test). Scale bars, $10 \mu \mathrm{m}$.

Fig. 7 | SCAP regulates CARTS biogenesis in a cholesterol-dependent manner. a, Schematic representation of the SCAP topology, where the Y234 and Y298 residues, loop1 and loop7, and WD (tryptophan-aspartate) repeats are indicated. b, Establishment of shSCAP HeLa cells stably expressing the hamster SCAP WT, Y234A, or Y298C mutants. Cell lysates were immunoblotted (IB) with the indicated antibodies. Ham, hamster; Hum, human. c, Recovery of PI4P turnover on expression of hamster SCAP WT, but not of Y234A or Y298C. The graph shows determination of the Golgi PI4P levels in the indicated cells. Data are mean \pm s.e.m. (Parental HeLa: $n=69$ cells; shSCAP: $\mathrm{n}=83$ cells; shSCAP + WT: $\mathrm{n}=78$ cells; shSCAP + Y234A: $\mathrm{n}=75$ cells; shSCAP + Y298C: $\mathrm{n}=70$ cells, $* * * * P<0.0001$, one-way ANOVA multiple comparison test). d, Recovery of biogenesis of mKate2-FM4-PAUF-containing CARTS on expression of hamster SCAP WT, but not of Y234A or Y298C. The graph shows the number of mKate2-FM4-PAUF-containing CARTS in the indicated cells at $15 \mathrm{~min}$ after the temperature shift to $37^{\circ} \mathrm{C}$. Data are means \pm s.e.m. $(n=20$ cells per conditions, ${ }^{* * * *} P<0.0001$, one-way ANOVA multiple comparison test). Scale bars, $10 \mu \mathrm{m}$. 
Fig. 8 | SCAP/SREBP complex functions in CARTS biogenesis. a, Effects of SCAP knockdown and expression of the hamster SCAP WT, Y234A, or Y298C mutants on the expression levels of SREBP1 and SREBP2. Cell lysates were immunoblotted (IB) with the indicated antibodies. The precursor $(\mathrm{P})$, but not mature $(\mathrm{M})$ forms of SREBP2 were detected in parental HeLa cells and shSCAP HeLa cells stably expressing the hamster SCAP WT, Y234A, or Y298C mutants. Expression levels of LDLR and HMGR were slightly increased by expression of hamster SCAP WT, Y234A, or Y298C mutants. b, Interactions of FLAG-SREBP1a and FLAG-SREBP2 with Myc-OSBP, Sac1, and VAP-A, but not with RTN-4B and Bap31 in HEK 293T cells. Cell lysates were subjected to immunoprecipitation with an anti-FLAG M2 affinity gel, and the cell lysates (Input) and immunoprecipitates (IP) were immunoblotted (IB) with the indicated antibodies. Asterisk denotes nonspecific bands. c, Interactions of FLAGSREBP1a and FLAG-SREBP2 with GFP-SCAP, Myc-OSBP, Sac1, and VAP-A, but not with RTN-4B and Bap31. Asterisk denotes nonspecific bands. d, siRNA-mediated knockdown of SREBP1 and/or SREBP2 in HeLa cells, monitored by Western blotting. Asterisks denote nonspecific bands. e, Biogenesis of mKate2-FM4-PAUF-containing CARTS in control, SREBP1, and SREBP2 knockdown cells. The graph shows the number of mKate2-FM4-PAUF-containing CARTS in the indicated cells at $15 \mathrm{~min}$ after the temperature shift to $37^{\circ} \mathrm{C}$. Data are means \pm s.e.m. $(n=11$ cells per conditions, ${ }^{*} P<0.05,{ }^{* * * *} P<0.0001$, one-way ANOVA multiple comparison test). Scale bar, 10 $\mu \mathrm{m}$.

Fig. 9 | Working model for the facilitation of CARTS biogenesis by the SCAP/SREBP complex at ER-Golgi contact sites. When the ER cholesterol (Chol) level is low, SCAP escorts SREBP transcription factors from the ER to the Golgi complex for cholesterol synthesis and uptake (left panel, blue arrows). When the ER contains sufficient levels of cholesterol, a complex of cholesterol-bound SCAP and SREBP interacts with the VAP/OSBP complex via Sac1, and functions in the countertransport of ER cholesterol and Golgi PI4P at ER-Golgi contact sites to promote CARTS biogenesis at the TGN domains immediately adjacent to the ER contacts sites 
(left panel, red arrows, and right panel). At these TGN domains, ceramide (Cer) transported by the VAP/CERT complex is metabolized together with phosphatidylcholine (PC) to SM and DAG. SM assembles with cholesterol into lipid nanodomains for processing and sorting of cargoes, while DAG recruits PKD for membrane fission, leading to CARTS biogenesis.

\section{Supplementary Fig. 1 | Characterization of the interaction of FLAG-Sac1 with} Myc-OSBP PH-FFAT mutant, and the interaction of stably-expressed FLAGSCAP with Sac1. a, Interactions of FLAG-Sac1 with VAP-A and Myc-OSBP WT, but not with the PH-FFAT mutant in HEK 293T cells. Cell lysates were subjected to immunoprecipitation with an anti-FLAG M2 affinity gel, and the cell lysates (Input) and immunoprecipitates (IP) were immunoblotted (IB) with the indicated antibodies. The interaction of FLAG-Sac1 with VAP-A was enhanced by coexpression with MycOSBP WT, but not with PH-FFAT. Single and double asterisks denote degraded MycOSBP fragments and the immunoglobulin heavy chain, respectively. b, A schematic representation of the SCAP interactions with VAP, OSBP, and Sac1. c, Establishment of a HeLa stable cell line expressing FLAG-SCAP. Cell lysates were immunoblotted with indicated antibodies. One of two SCAP antibodies recognizes both human (Hum) and hamster (Ham) SCAP and the other is hamster-specific. Asterisks denote nonspecific bands. d, Interaction of stably-expressed FLAG-SCAP with Sac1, but not with RTN-4B and Bap31. VAP-A was detected as a very faint band in the immunoprecipitate of the stable cell line (lane 4).

\section{Supplementary Fig. 2 | BiFC visualization of VAP-A, OSBP, and Sac1 interactions} at ER-Golgi contact sites. a,b, No BiFC signal in HeLa cells with single expression of Venus N-terminal fragment (Vn)-fused proteins (a) or Venus C-terminal fragment (Vc)-fused proteins (b). c,d, BiFC visualization of OSBP/VAP-A (c) and OSBP/Sac1 (d) interactions at ER-Golgi contact sites. The coexpression of Vn-OSBP (WT or FF/AA) with Vc-VAP-A (c) or Vc-Sac1 (d) was visualized with an anti-GFP antibody. The BiFC signal was enhanced by treatment of cells with $2 \mu \mathrm{g} / \mathrm{mL} 25-\mathrm{HC}$ for $2.5 \mathrm{~h}$. 
Vn-OSBP FF/AA showed a reduced BiFC signal, compared with the WT. Scale bars, $10 \mu \mathrm{m}$.

Supplementary Fig. 3 | Sterol-dependent localization of SCAP. a,b, Localization of stably expressed GFP-SCAP in HeLa cells with or without cholesterol depletion (Chol Depl) for $3 \mathrm{~h}$ or treated with $2 \mu \mathrm{g} / \mathrm{mL} 25-\mathrm{HC}$ for $2.5 \mathrm{~h}$. Accumulation of GFP-SCAP in GM130 (cis-Golgi matrix protein)- and Mannosidase II (cis/medial-Golgi marker enzyme)-positive membranes upon cholesterol depletion for $3 \mathrm{~h}(\mathbf{b})$. $\mathrm{N}$, nucleus. c, Proteolytic activation of SREBP2 upon cholesterol depletion, monitored by Western blotting with an anti-SREBP2 antibody. Arrowheads indicate the precursor $(\mathrm{P})$ and mature (M) forms of SREBP2. Asterisk denotes nonspecific bands. d, Localization of the stably-expressed hamster SCAP WT, Y234A, and Y298C mutants in shSCAP HeLa cells with or without cholesterol depletion for $3 \mathrm{~h}$. Merged images for the hamster SCAP WT, Y234A or Y298C (green) and the cis/medial-Golgi marker GPP130 (magenta) of the boxed areas are shown in the insets. Scale bars, $10 \mu \mathrm{m}$.

\section{Supplementary Fig. 4 | Effects of knockdown of ER-Golgi contact site components} on PI4P turnover, and visualization of VAP-A/OSBP complex at ER-Golgi contact sites . a, PI4P staining in control (Cont), VAP-A/B, CERT/OSBP, and Sac1 knockdown HeLa cells. b, HeLa cells stably coexpressing Vn-OSBP and Vc-VAP-A were treated with or without $2 \mu \mathrm{g} / \mathrm{mL} 25-\mathrm{HC}$ for $2.5 \mathrm{~h}$. The coexpression of Vn-OSBP and Vc-VAPA was visualized with an anti-GFP antibody. Scale bars, $10 \mu \mathrm{m}$.

\section{Supplementary Fig. 5 | Lipid transfer complexes at ER-Golgi contact sites are} required for GPI-anchored protein transport from the TGN to the PM. a, mKate2FM4-GPI transport from the ER to the PM via the Golgi complex in control (Cont), OSBP, and CERT/OSBP knockdown cells. The cells were incubated at $37^{\circ} \mathrm{C}$ with the $\mathrm{D} / \mathrm{D}$ solubilizer and cycloheximide, and fixed at the indicated times. The graph shows the percentages of cells with mKate2-FM4-GPI at the ER, ER/ Golgi, Golgi/PM, or PM at the indicated times. The data shown are for a single representative experiment out of 
three performed (control siRNA: 30 min, $\mathrm{n}=240$ cells; $60 \mathrm{~min}, \mathrm{n}=230$ cells; $90 \mathrm{~min}$, $\mathrm{n}=233$ cells; OSBP siRNA: $30 \mathrm{~min}, \mathrm{n}=254$ cells; $60 \mathrm{~min}, \mathrm{n}=229$ cells; $90 \mathrm{~min}, \mathrm{n}=$ 238 cells; CERT/OSBP siRNA: 30 min, $\mathrm{n}=268$ cells; $60 \mathrm{~min}, \mathrm{n}=235$ cells; $90 \mathrm{~min}, \mathrm{n}$ $=245$ cells). $\mathbf{b}$, mKate2-FM4-GPI transport in the presence or absence of the MycOSBP PH-FFAT mutant. Asterisks denote cells coexpressing mKate2-FM4-GPI and Myc-OSBP PH-FFAT. Scale bars, $10 \mu \mathrm{m}$.

\section{Supplementary Video 1 | The biogenesis of mKate2-FM4-PAUF-containing}

CARTS at the TGN. HeLa cells expressing mKate2-FM4-PAUF were first incubated at $20^{\circ} \mathrm{C}$ with the $\mathrm{D} / \mathrm{D}$ solubilizer and cycloheximide for $45 \mathrm{~min}$. Images were acquired continuously after the temperature shift to $37^{\circ} \mathrm{C}$ with a time interval between frames of $30 \mathrm{sec}$ for $\sim 75 \mathrm{~min}$. $\mathrm{N}$, nucleus. 
bioRxiv preprint doi: https://doi.org/10.1101/679936; this version posted February 28, 2020. The copyright holder for this preprint (which

was not certified by peer review) is the author/funder, who has granted bioRxiv a license to display the preprint in perpetuity. It is made available under aCC-BY-NC-ND 4.0 International license.

Fig. 1

a

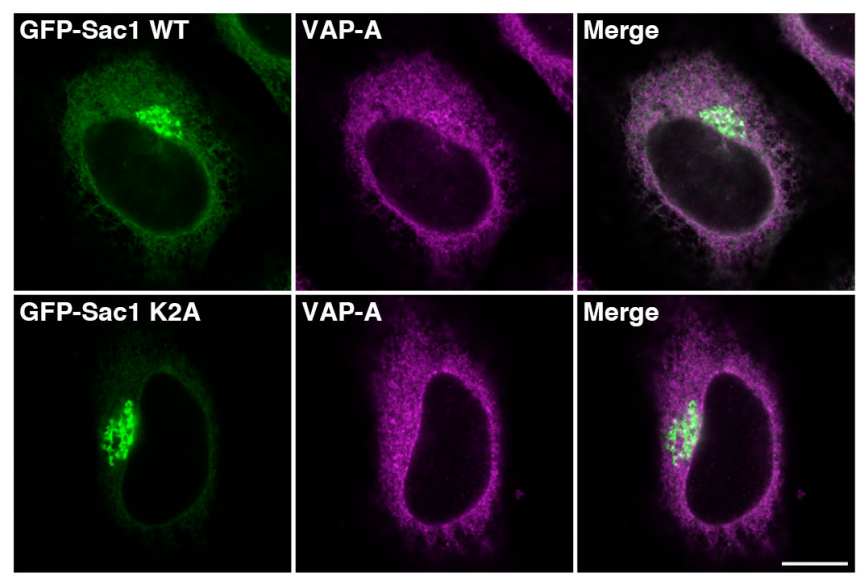

b
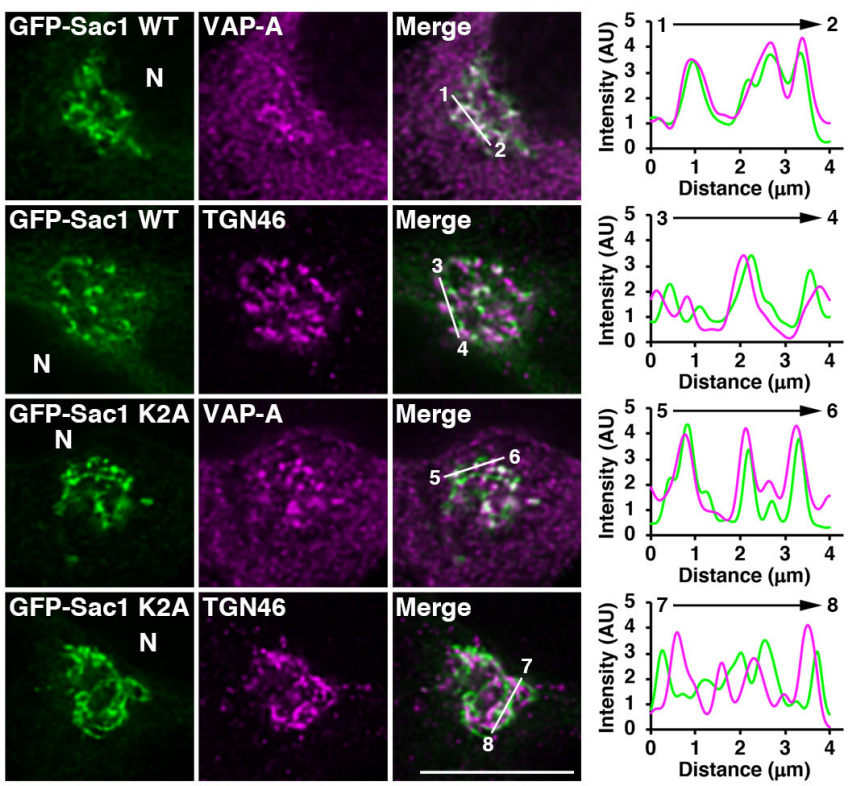

d

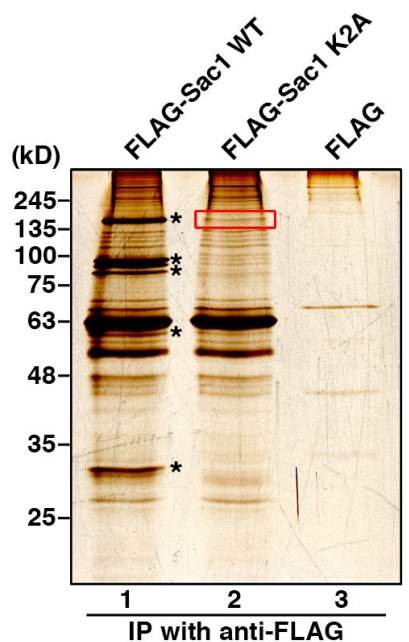

140-147: IISTTASK

148-155: TETPIVSK

OSBP

205-212: TELQNTLR

218-230: VEDLSTCNDLIAK

238-246: SLSELESLK

247-254: LPAESNEK

304-313: LEETLEQLAK

626-637: KVTGEVTDPSGK

627-637: VTGEVTDPSGK

729-736: WDEANAEK

757-768: ATEDGTPYDPYK
C
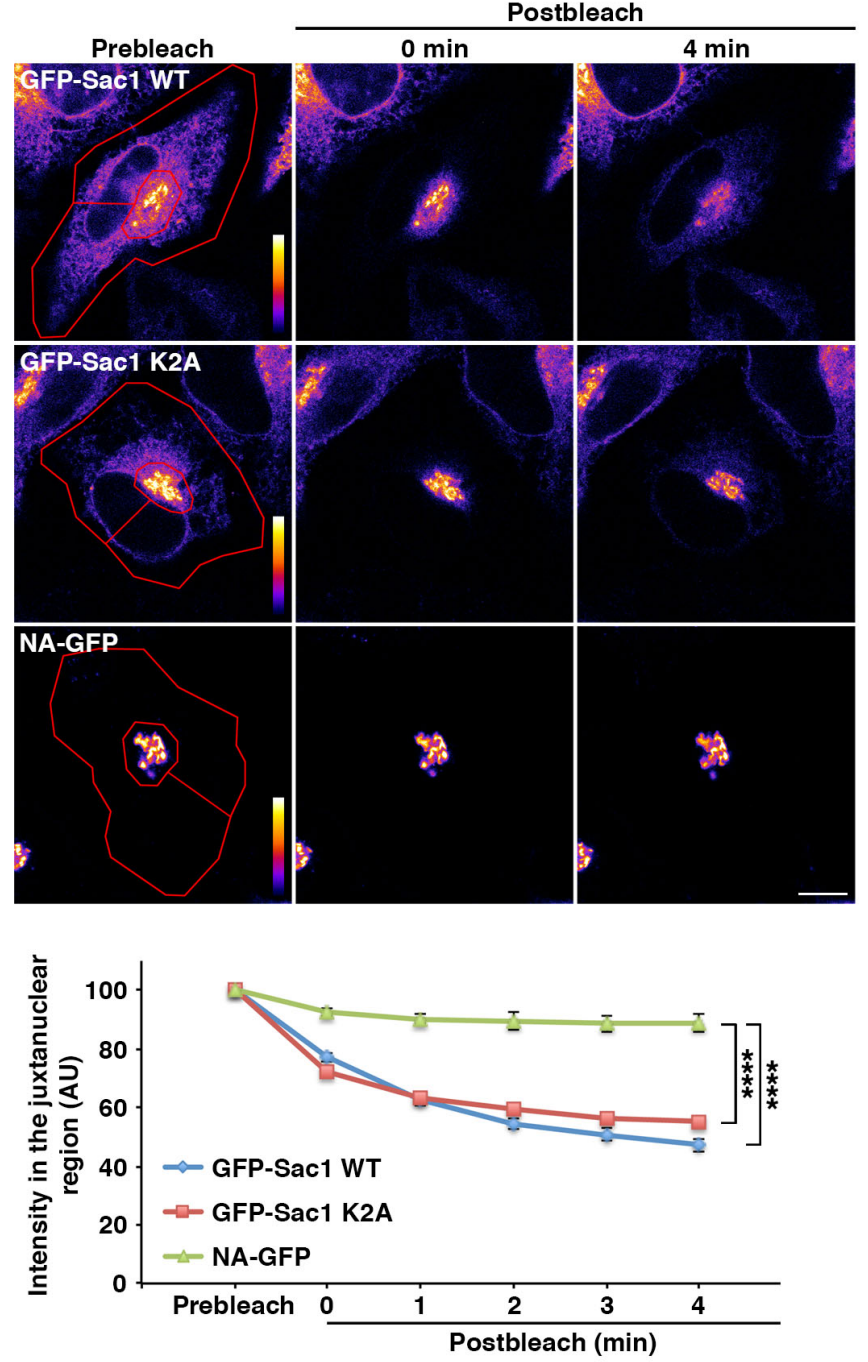

SCAP

47-62: LPLPGTGPVEFTTPVK

63-73: DYSPPPVDSDR

108-116: NLLAVDVFR

122-130: AFQLVEEIR

202-210: TLQTSATLK

211-219: DLLFGVPGK

367-378: SVVSTPVDLEVK

887-896: SSQPTQPEPR

1092-1102: LVTGSQDHTLR

201-216: TVQSNSPISALAPTGK

324-340: GATVLPANTPGNVGSGK

377-395: TGSNISGASSDISLDEQYK 
Fig. 2

a

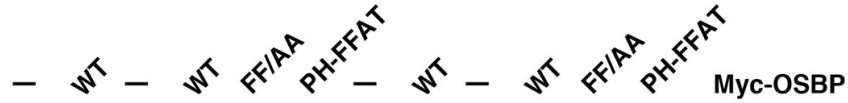

$$
\begin{aligned}
& --++++--++++ \text { FLAG-SCAP } \\
& \text { (kD) }++----++--- \text { FLAG }
\end{aligned}
$$
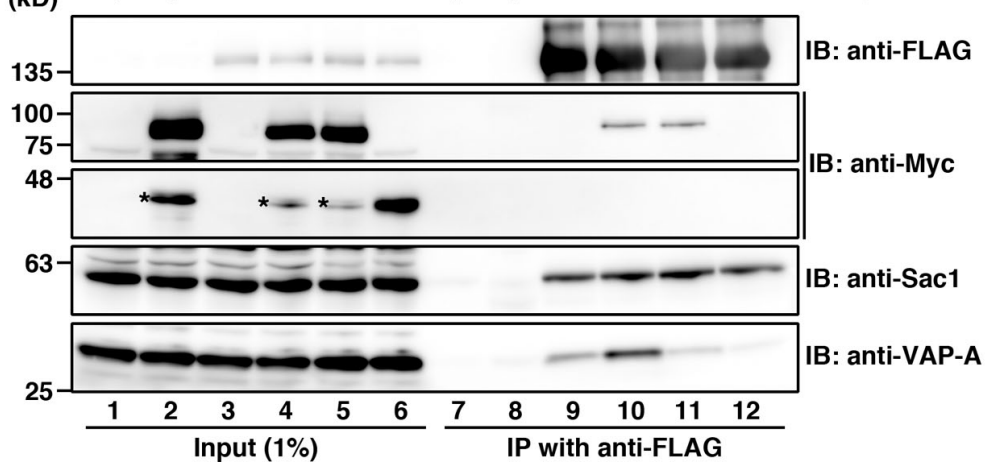

C

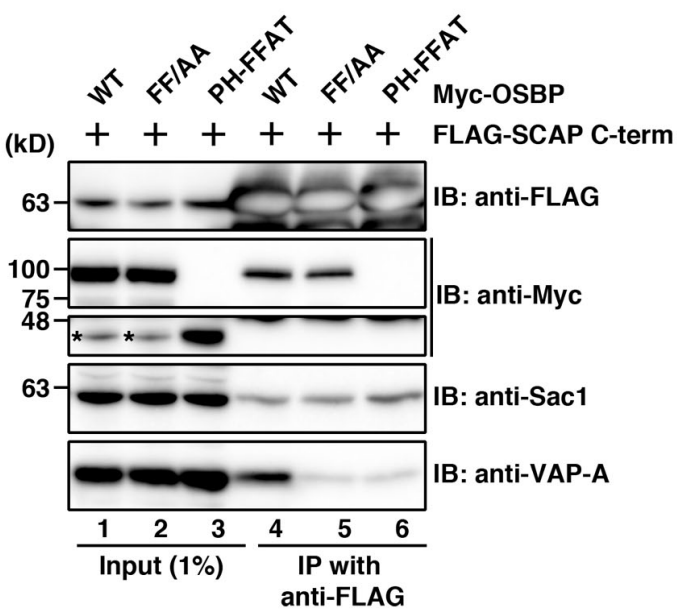

b

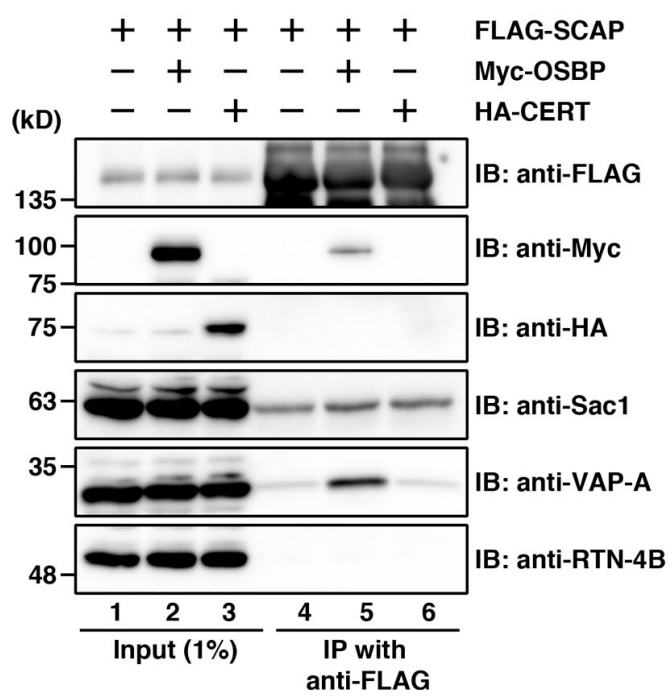

d

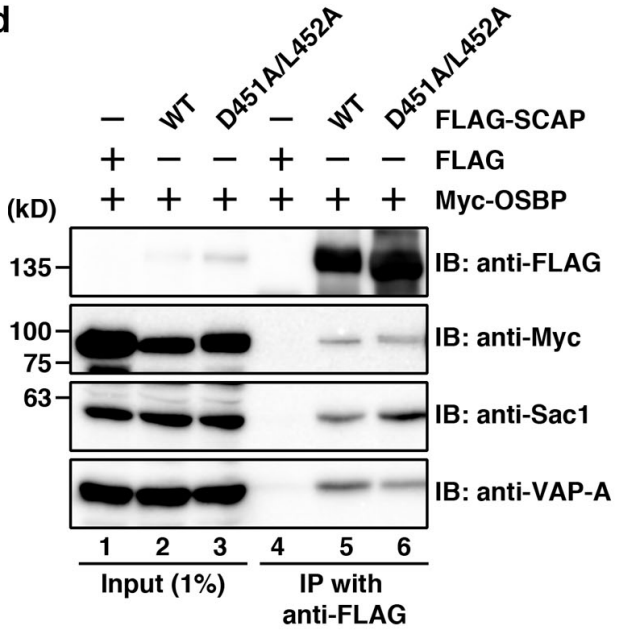

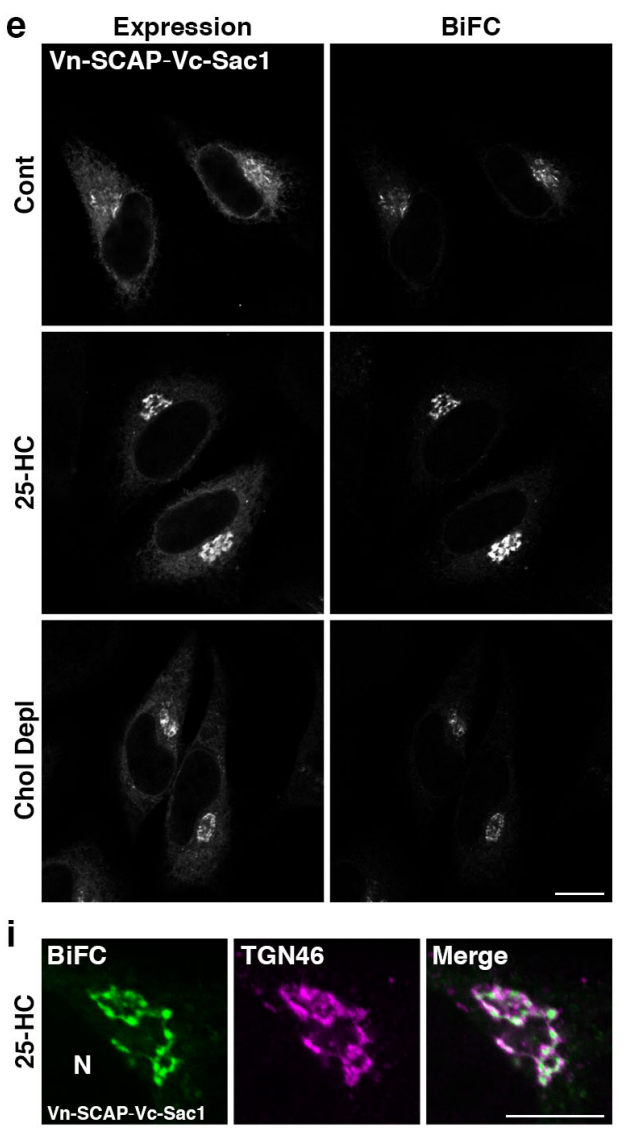

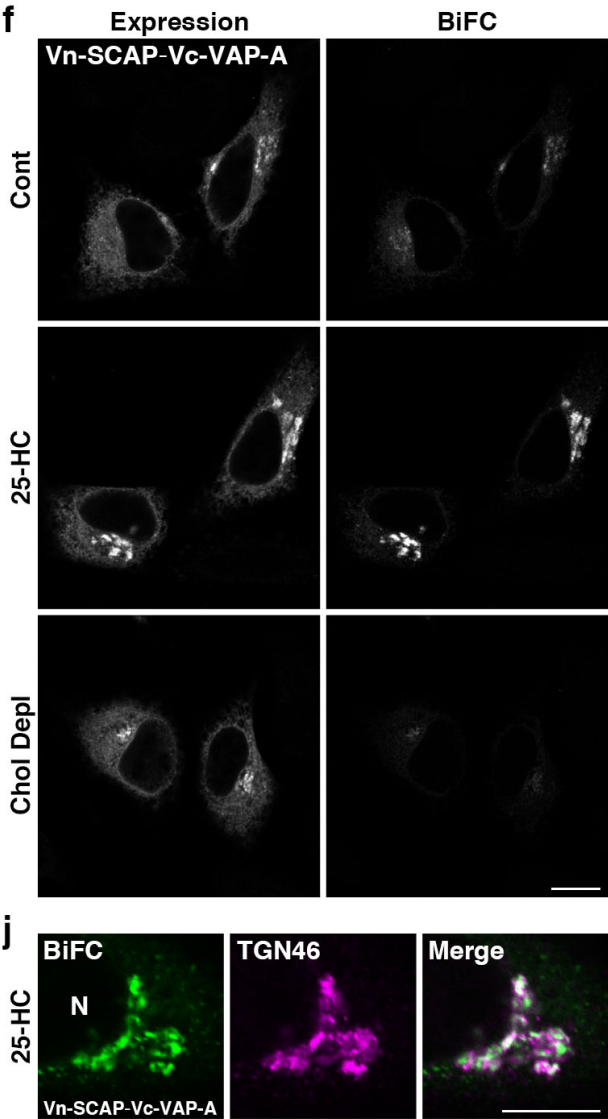

g

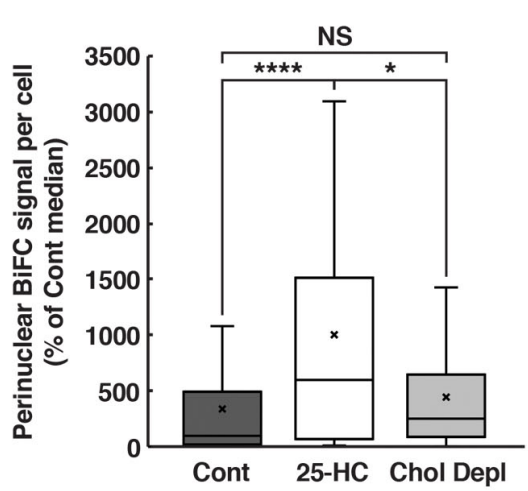

h

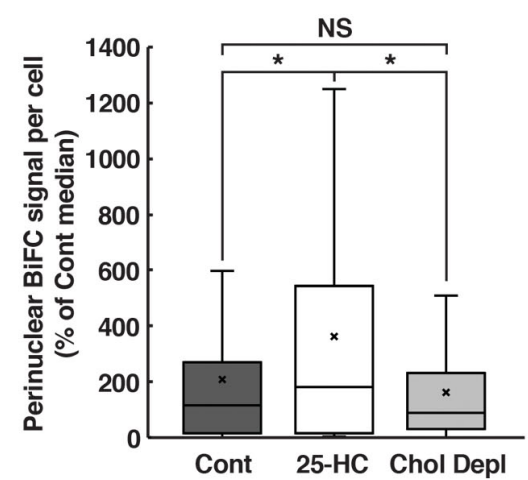


Fig. 3

a

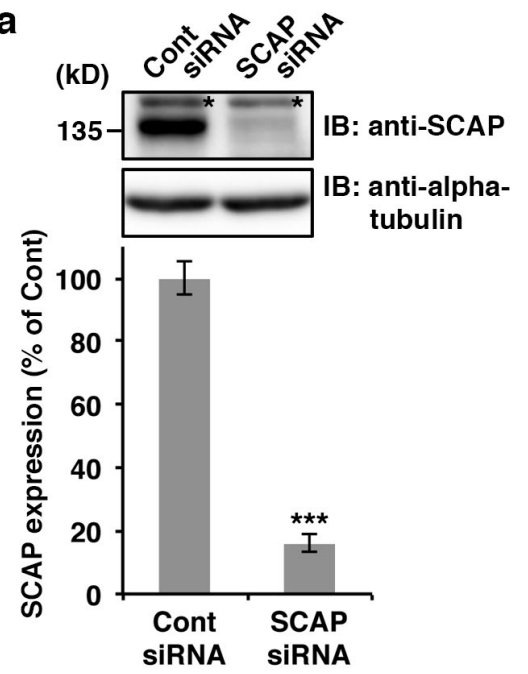

d

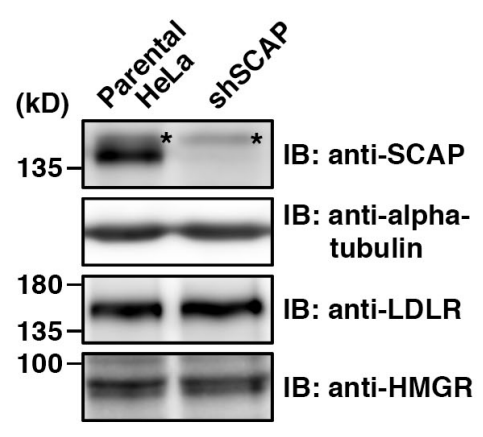

b

Cont SIRNA $\quad$ SCAP SIRNA

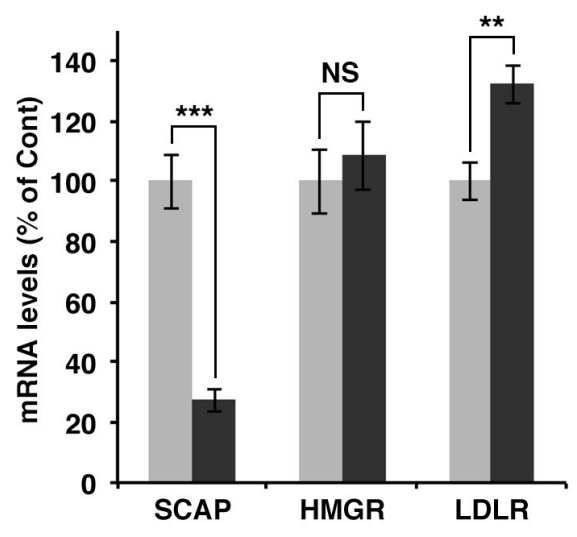

e

- Parental HeLa "shSCAP

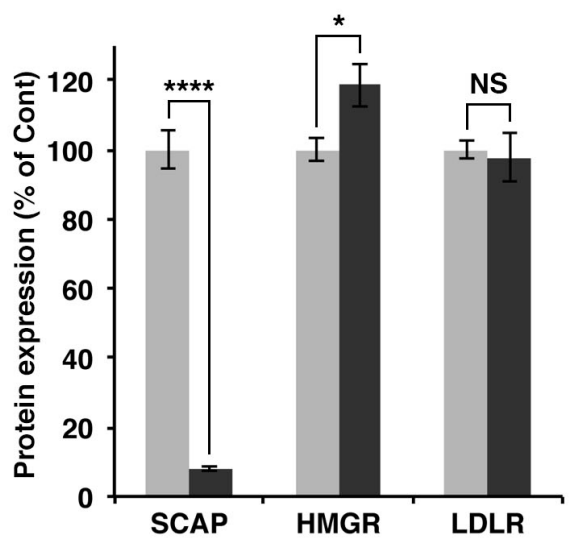

C

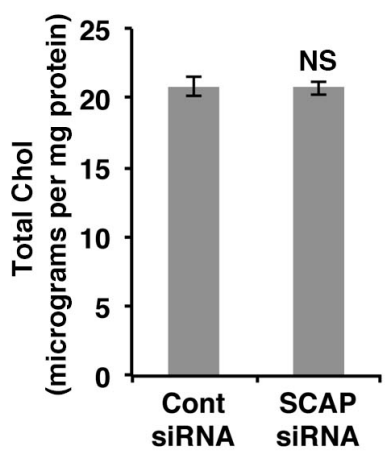

f

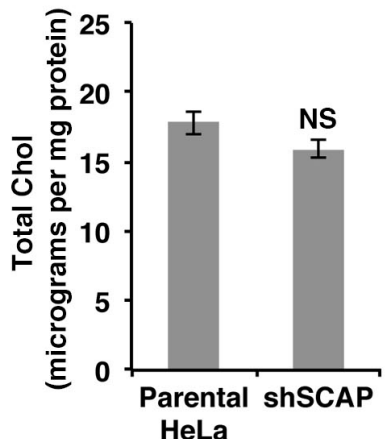


bioRxiv preprint doi: https://doi.org/10.1101/679936; this version posted February 28, 2020. The copyright holder for this preprint (which

was not certified by peer review) is the author/funder, who has granted bioRxiv a license to display the preprint in perpetuity. It is made available under aCC-BY-NC-ND 4.0 International license.

Fig. 4

a
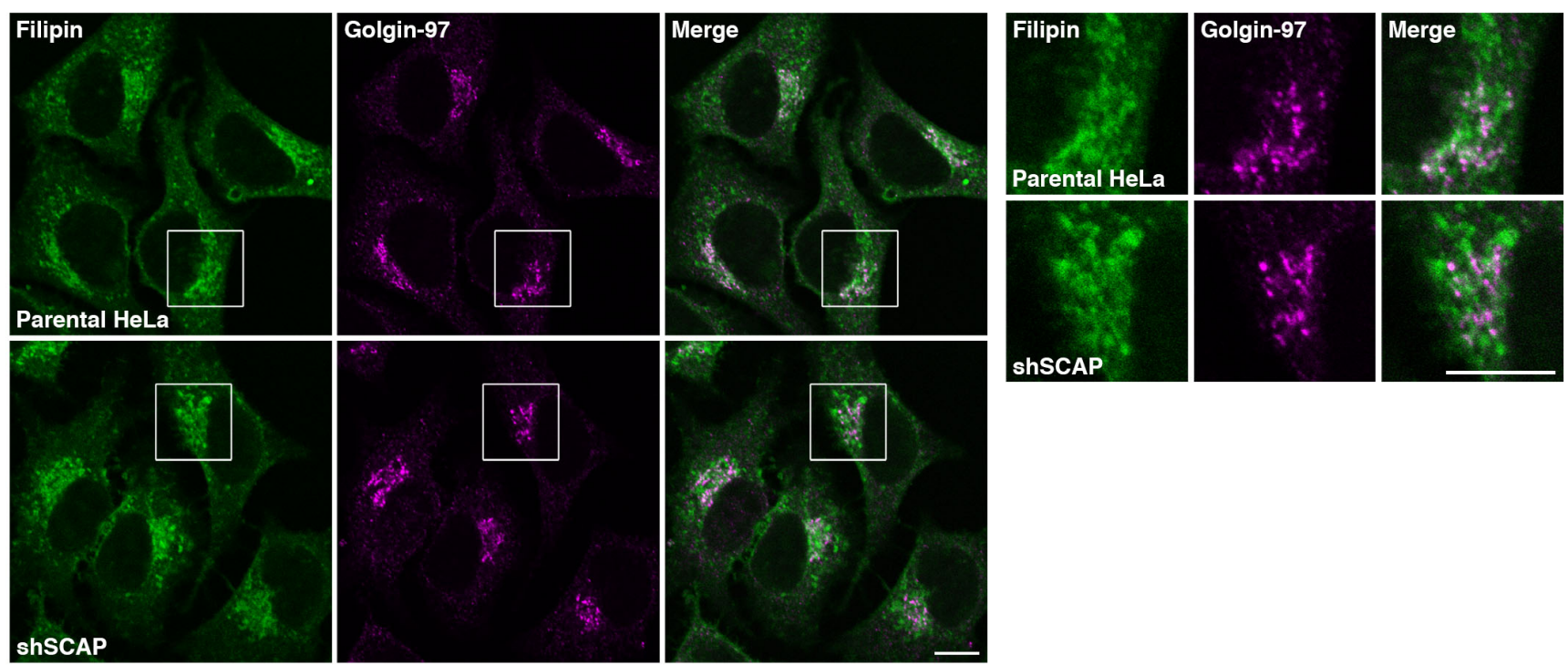

b

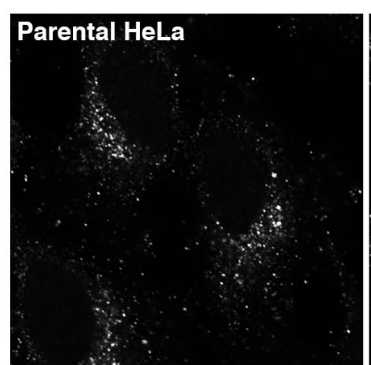

d
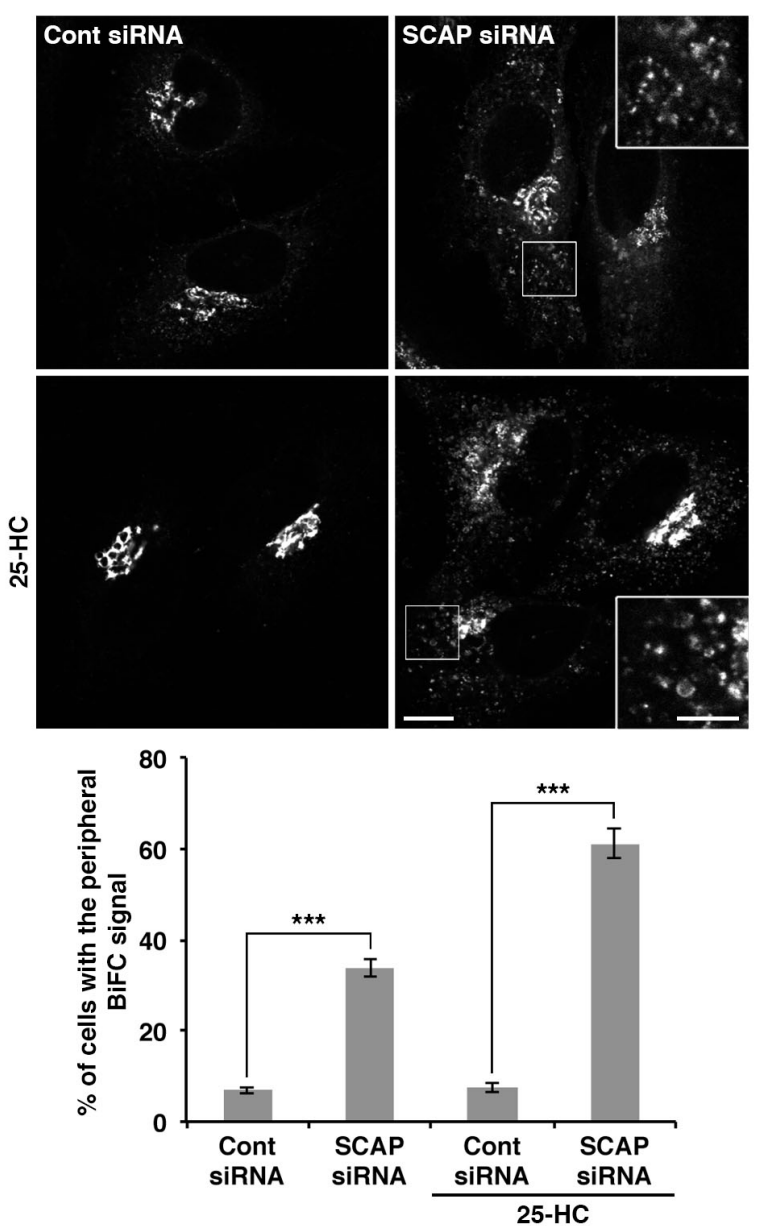
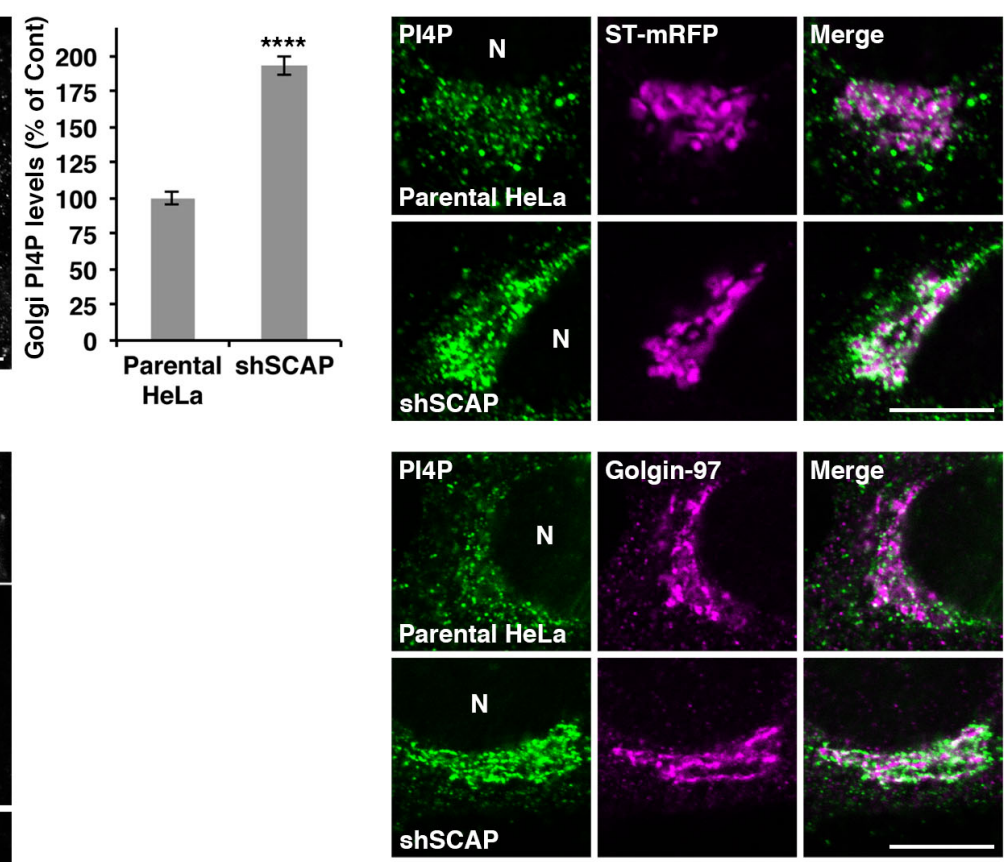

e

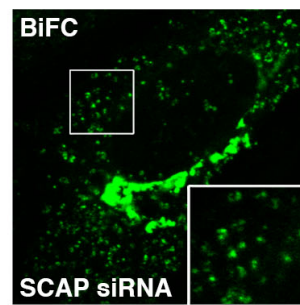

BiFC

TGN46

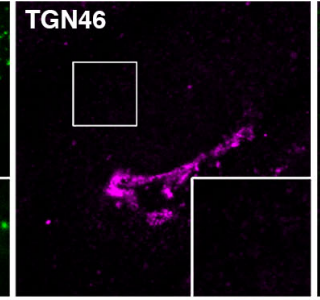

Merge

CD63
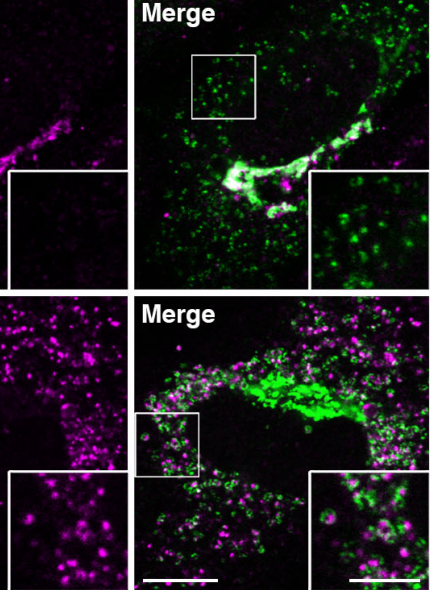

SCAP SIRNA 
Fig. 5

a
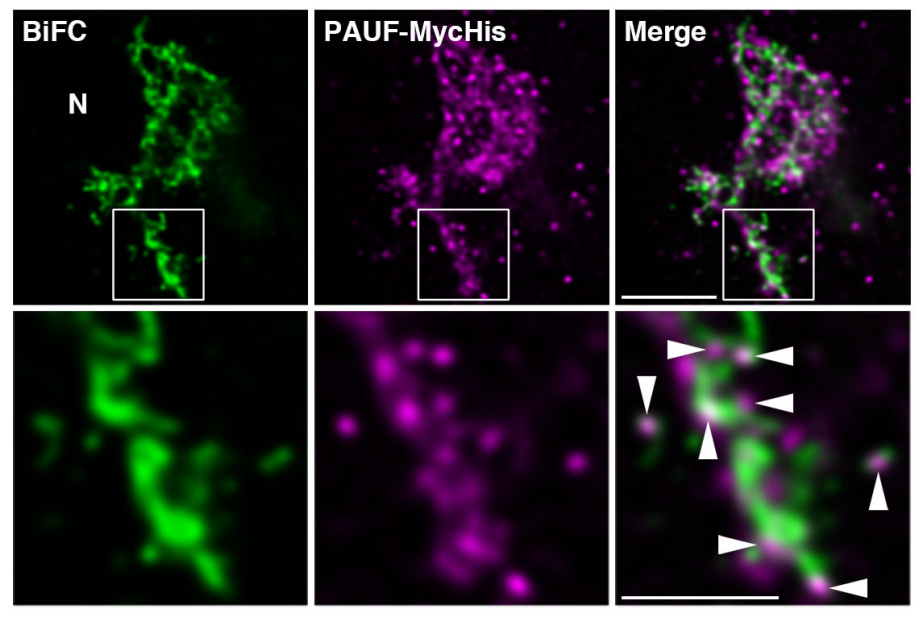

b

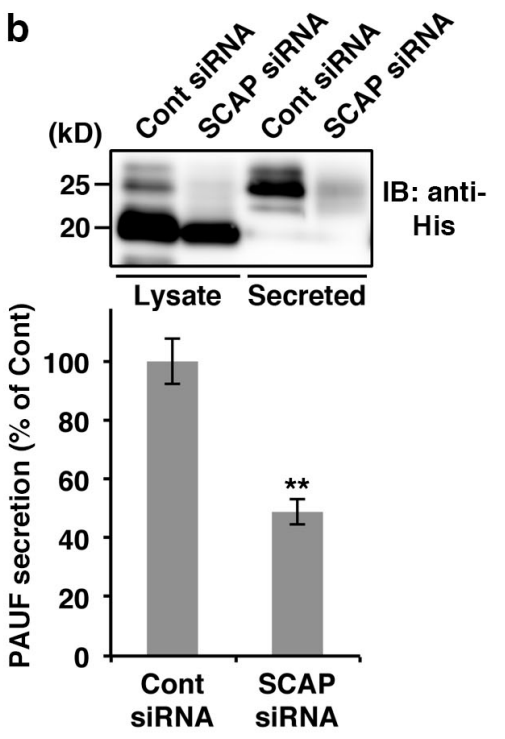

C
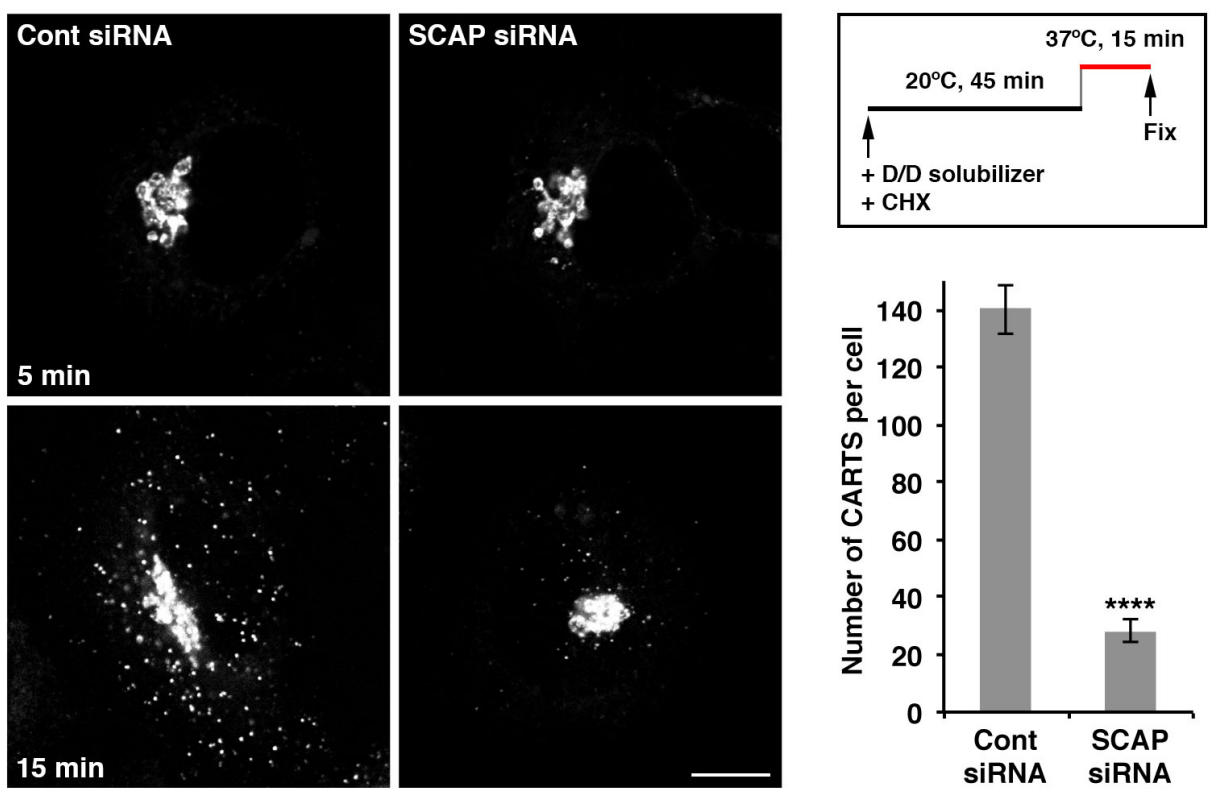


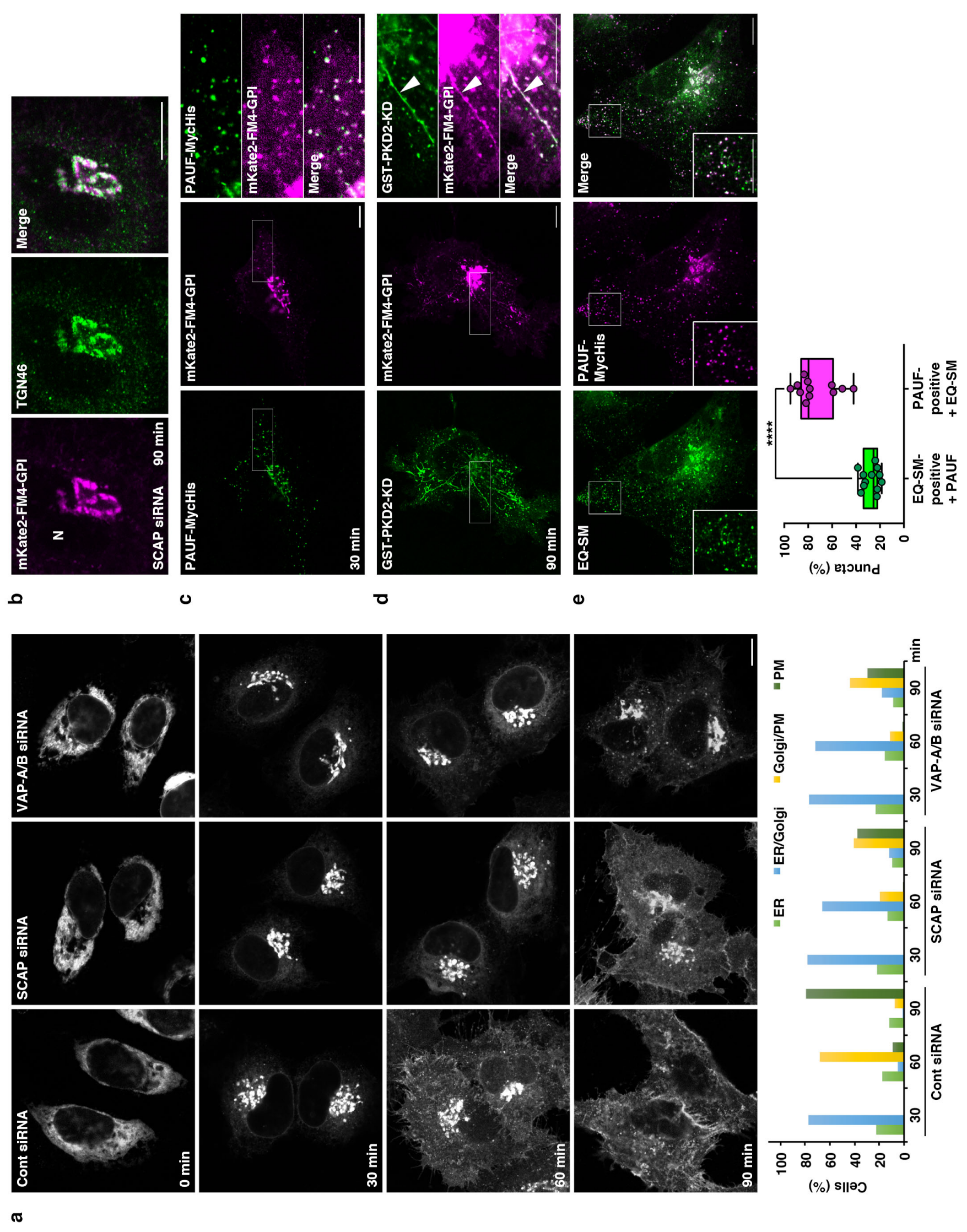


Fig. 7 available under aCC-BY-NC-ND 4.0 International license.

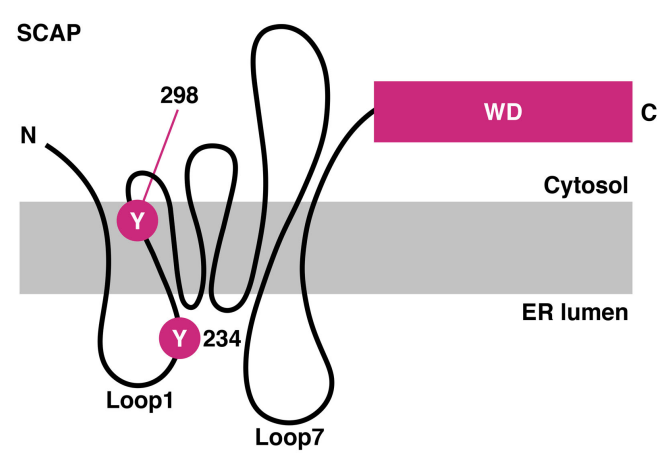

C
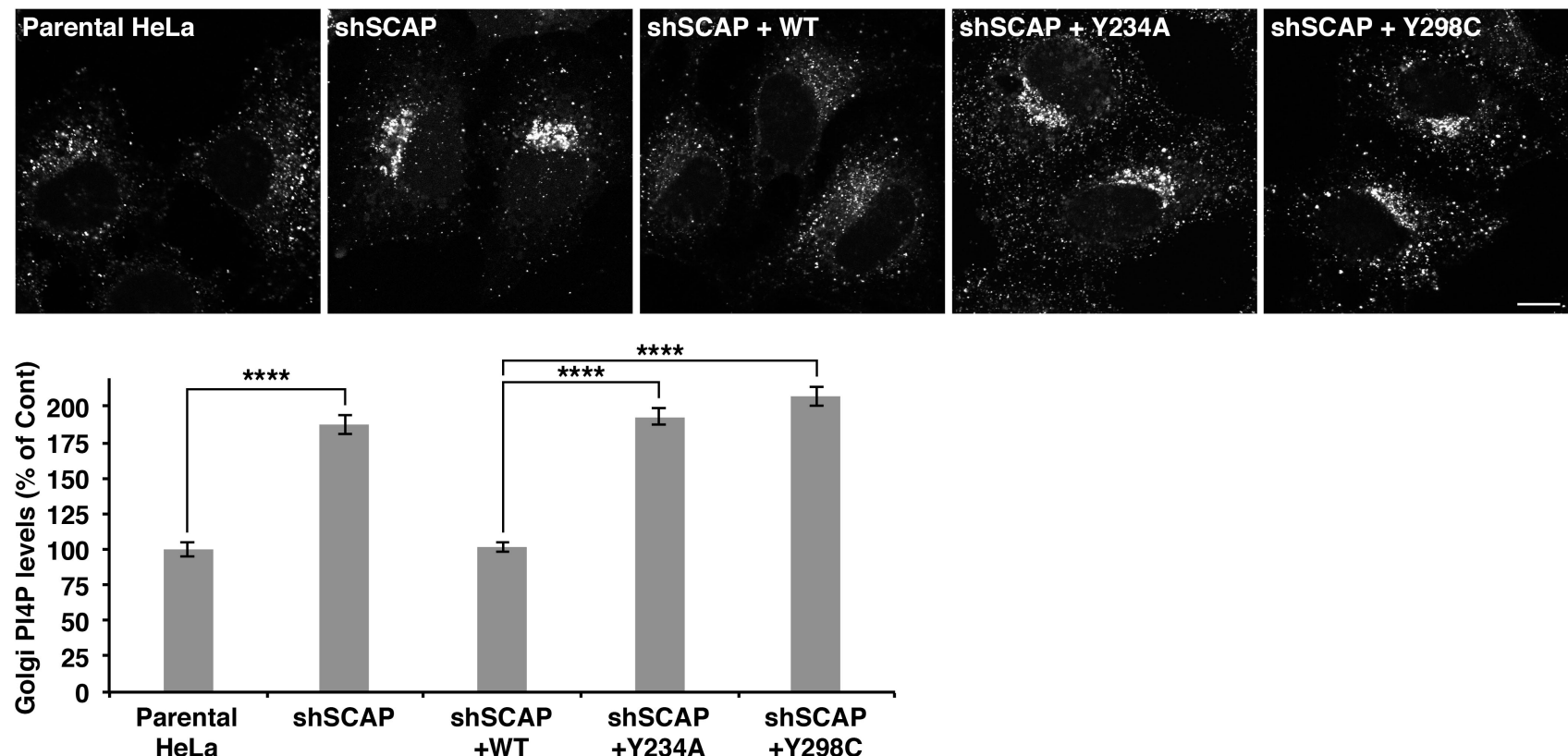

d
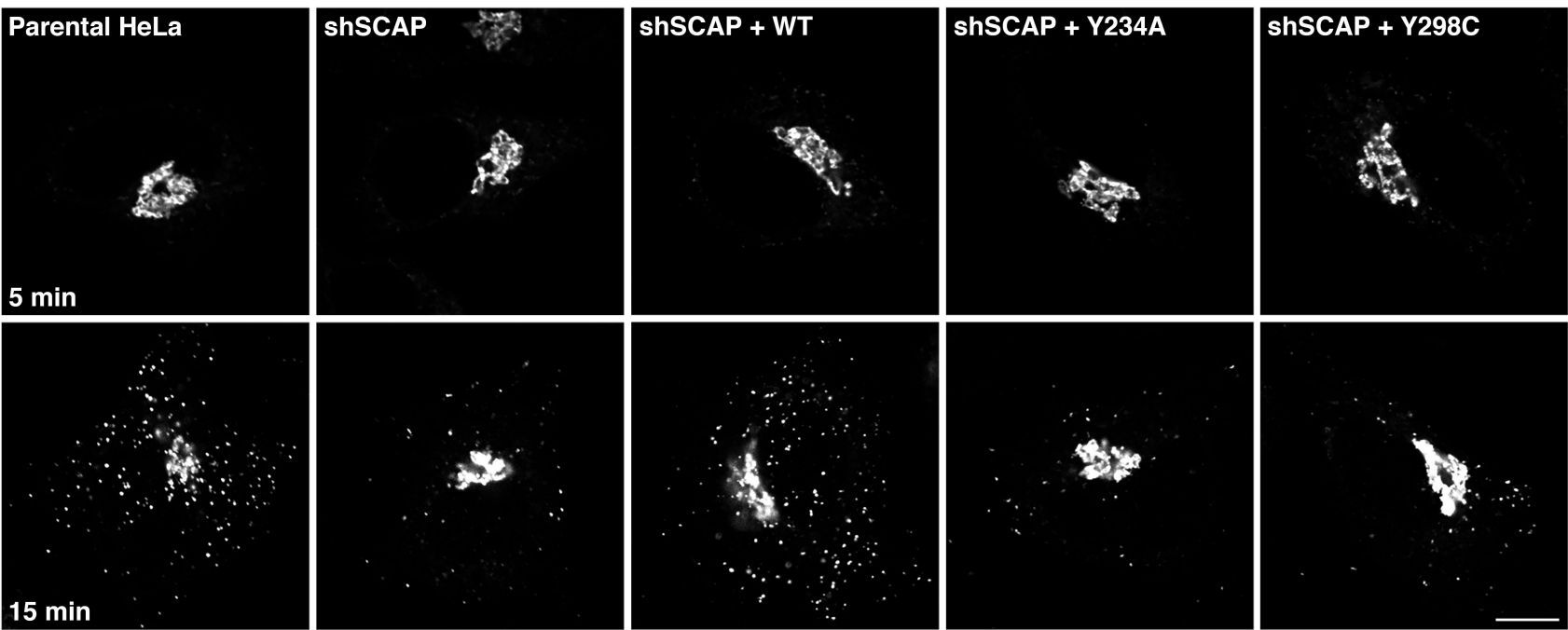

$15 \mathrm{~min}$
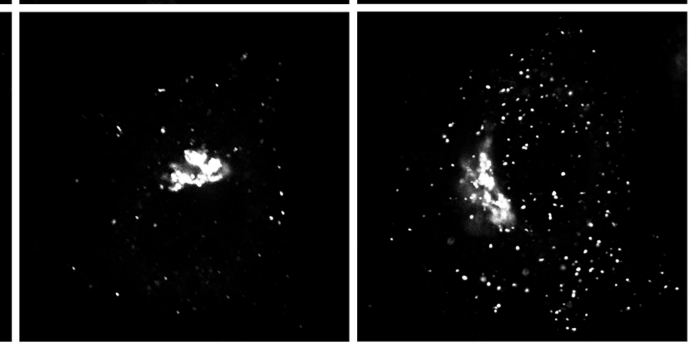
(Ham)

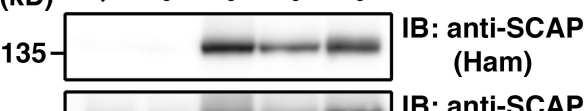

135 (Ham and Hum) IB: anti-alphatubulin

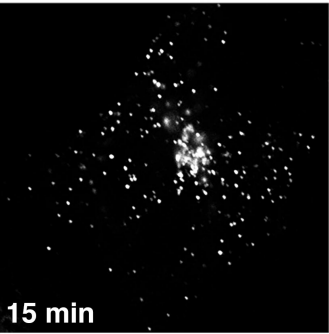

$\star \star \star \star$

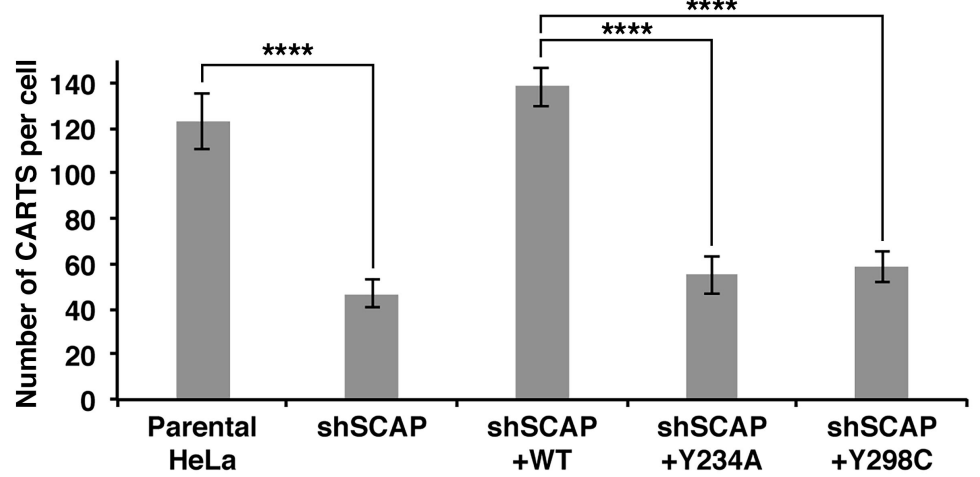




\section{Fig. 8}

a

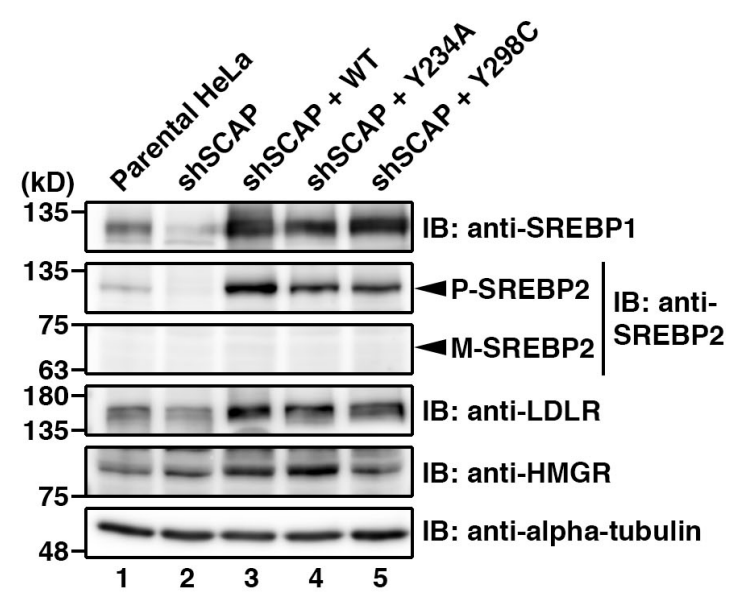

b

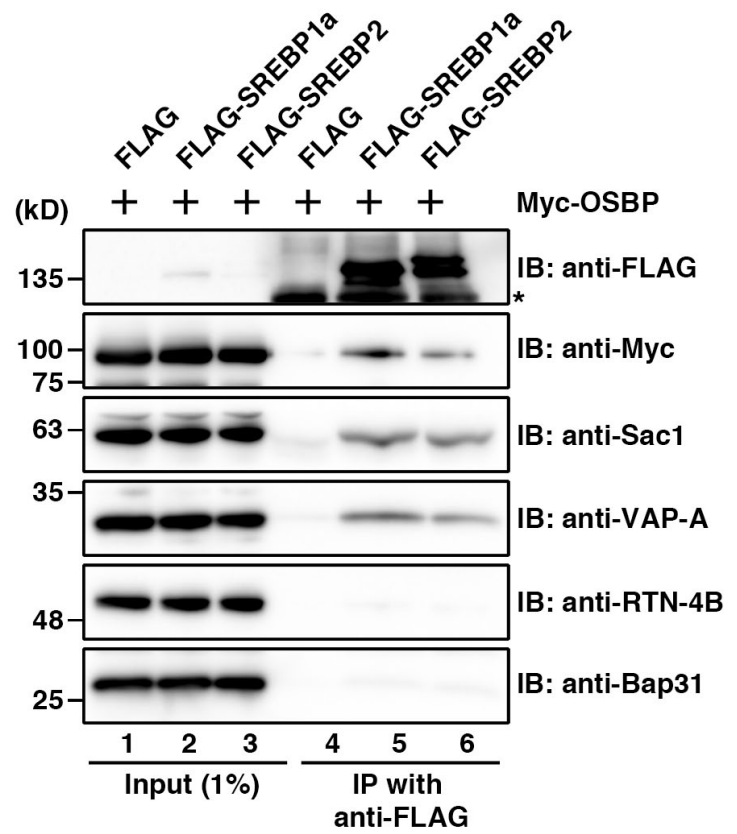

C

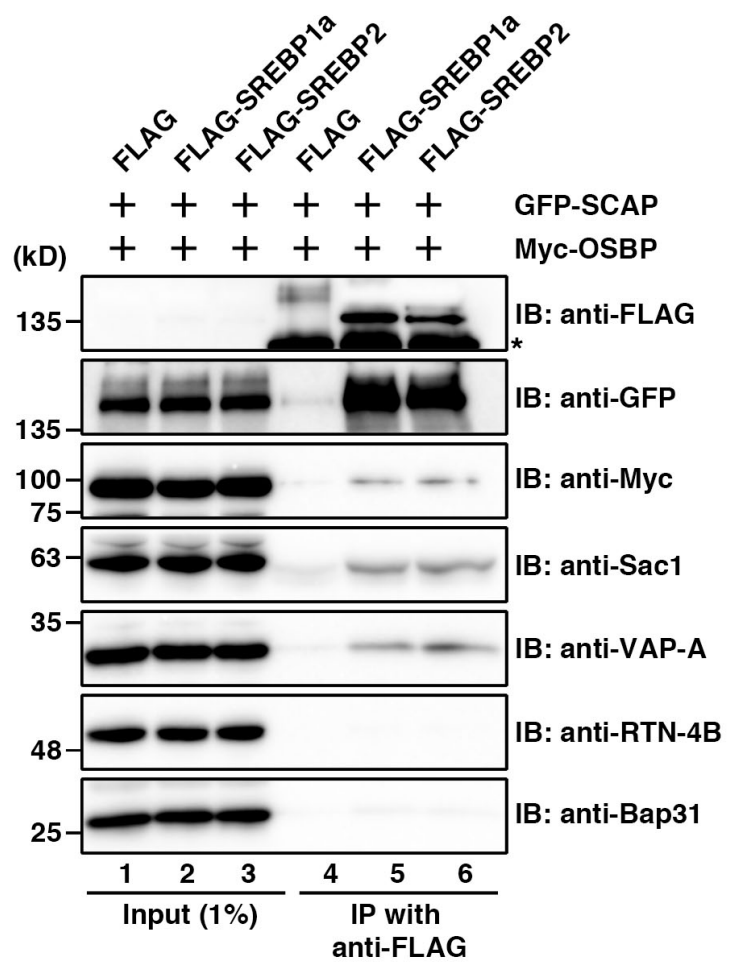

d

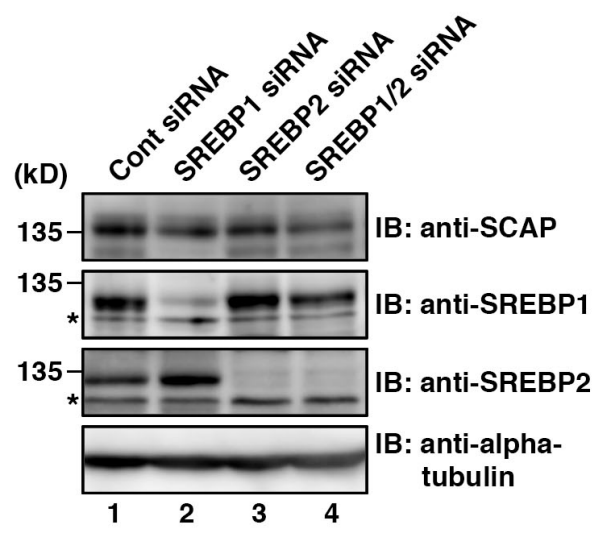

e
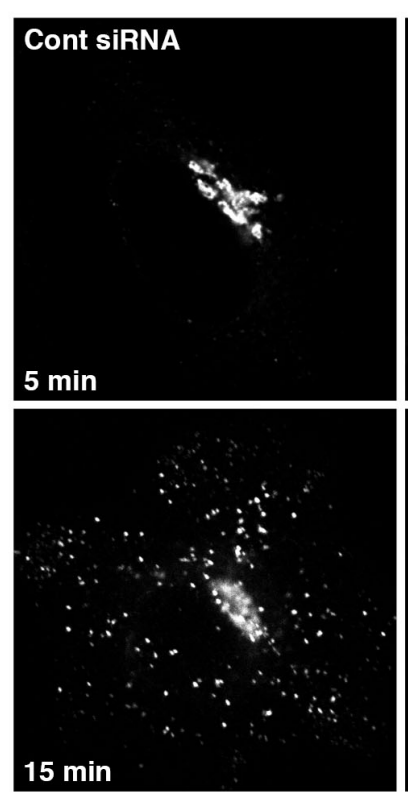
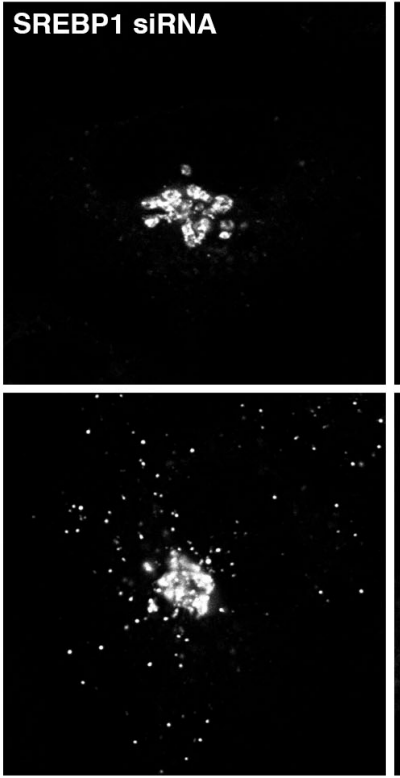
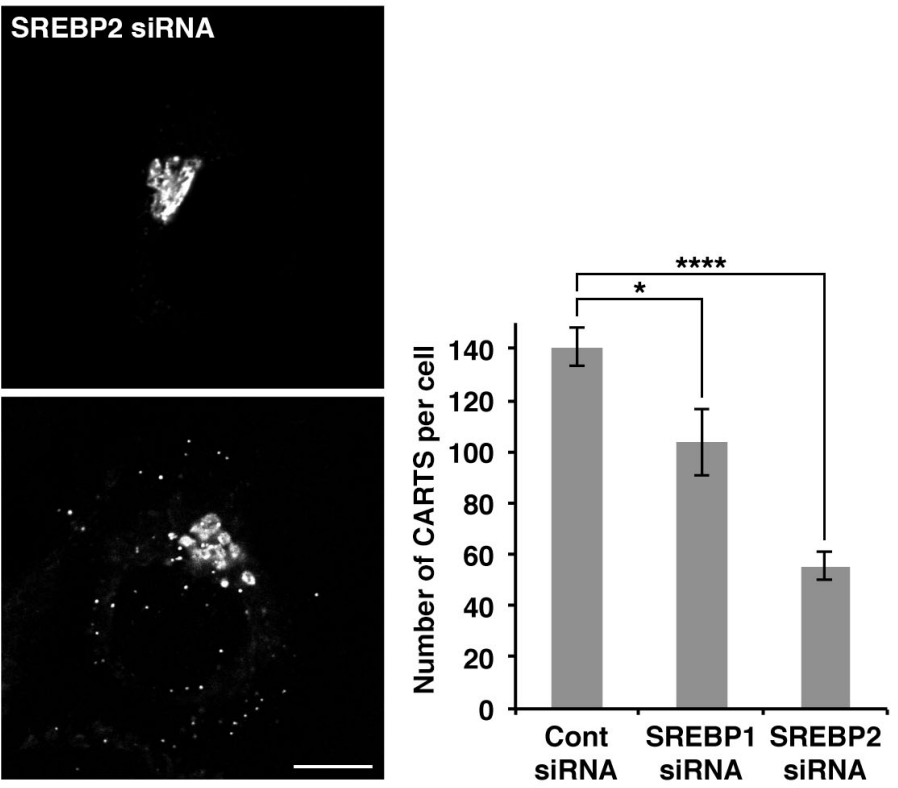


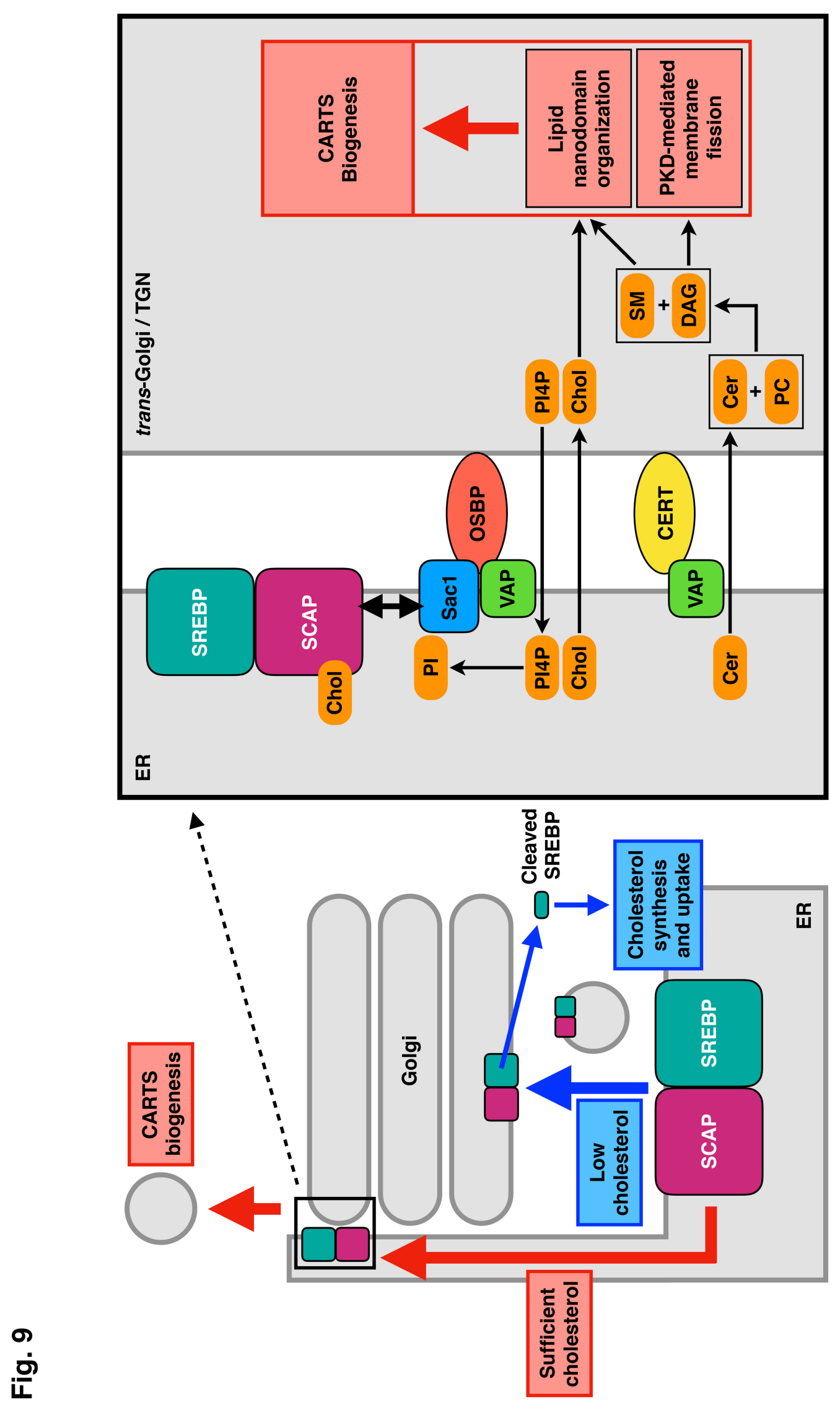




\section{Supplementary Fig. 1}

a

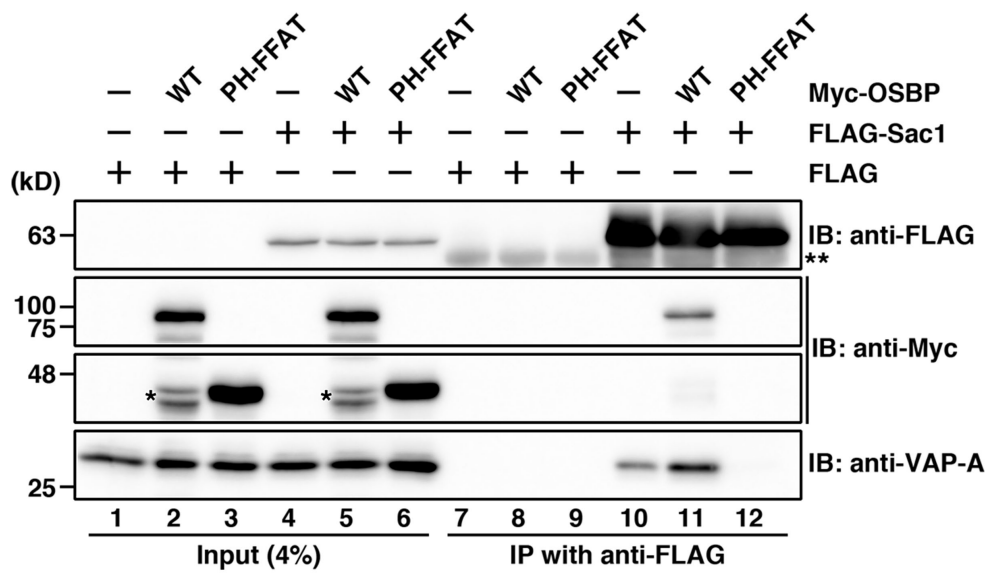

b

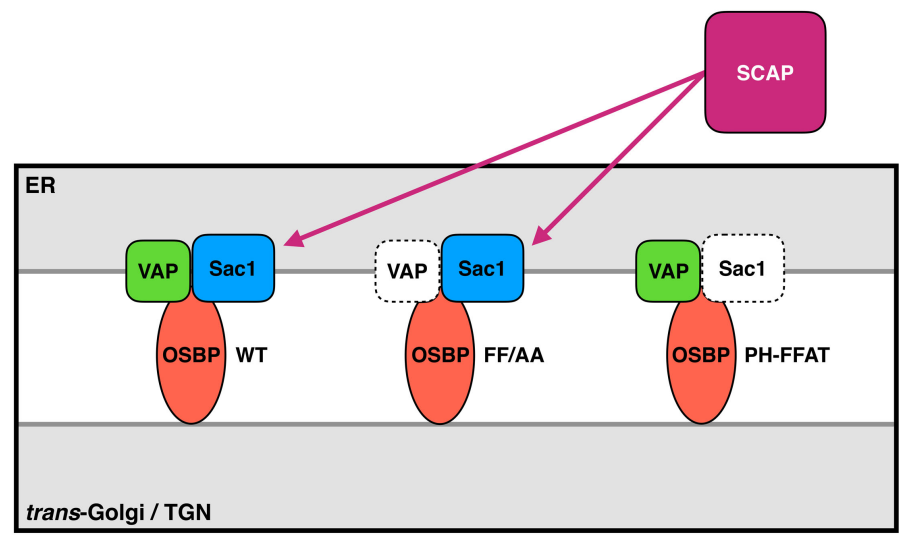

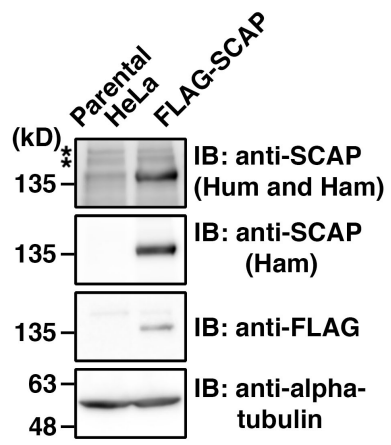

d

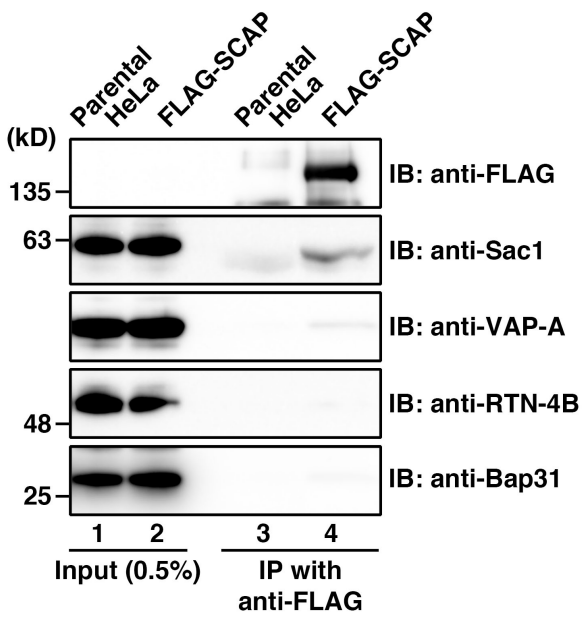


bioRxiv preprint doi: https://doi.org/10.1101/679936; this version posted February 28, 2020. The copyright holder for this preprint (which

was not certified by peer review) is the author/funder, who has granted bioRxiv a license to display the preprint in perpetuity. It is made available under aCC-BY-NC-ND 4.0 International license.

\section{Supplementary Fig. 2}

a

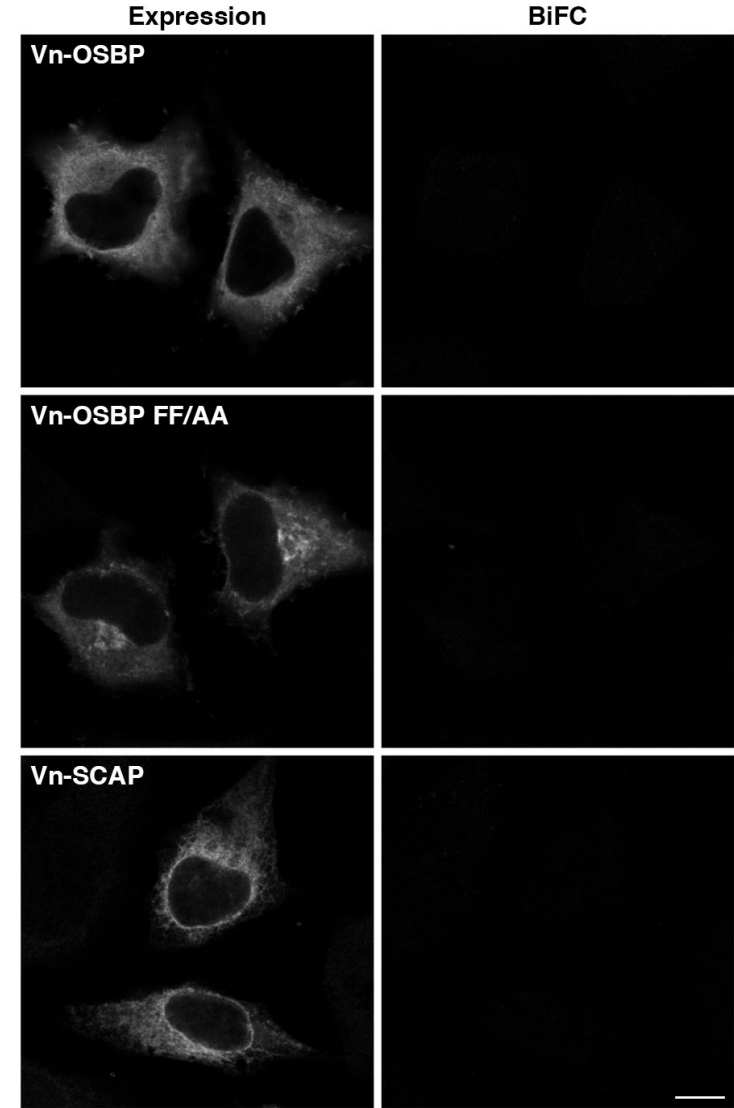

C

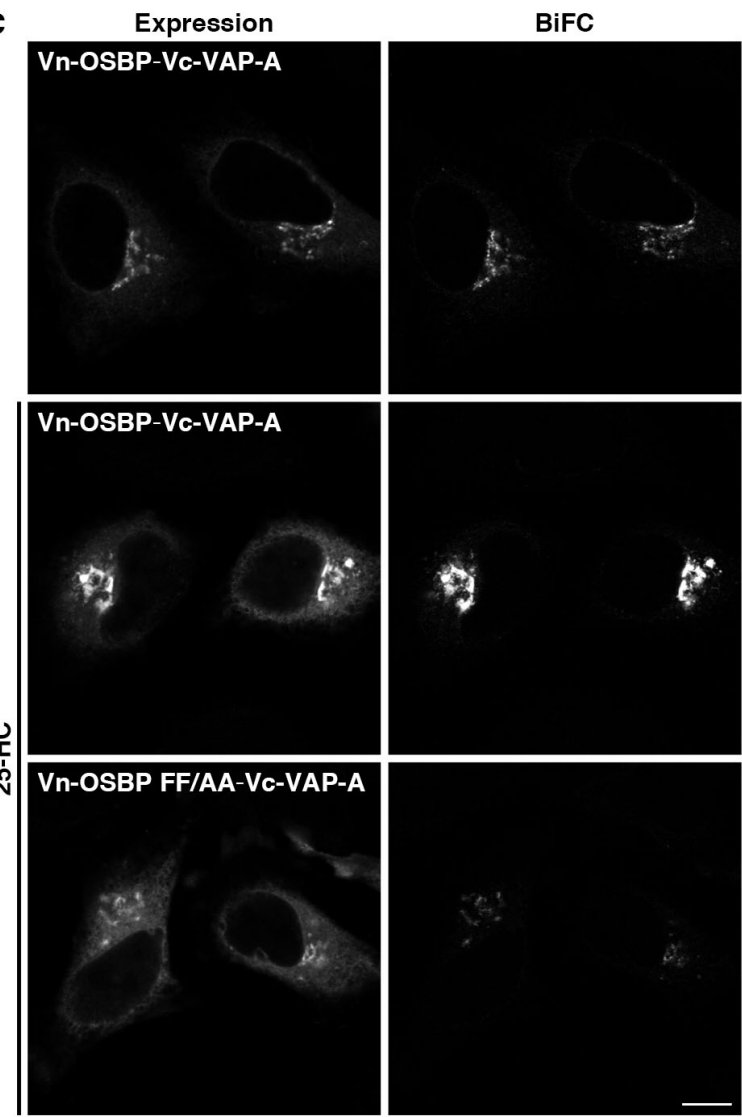

b
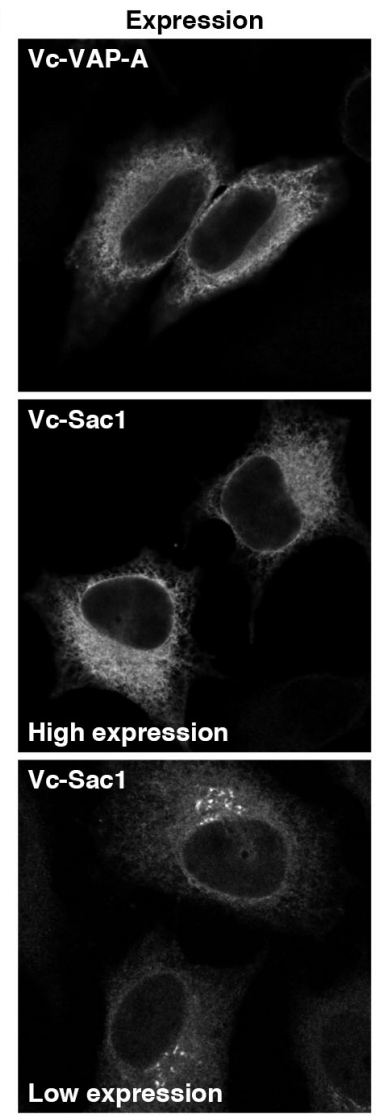

d

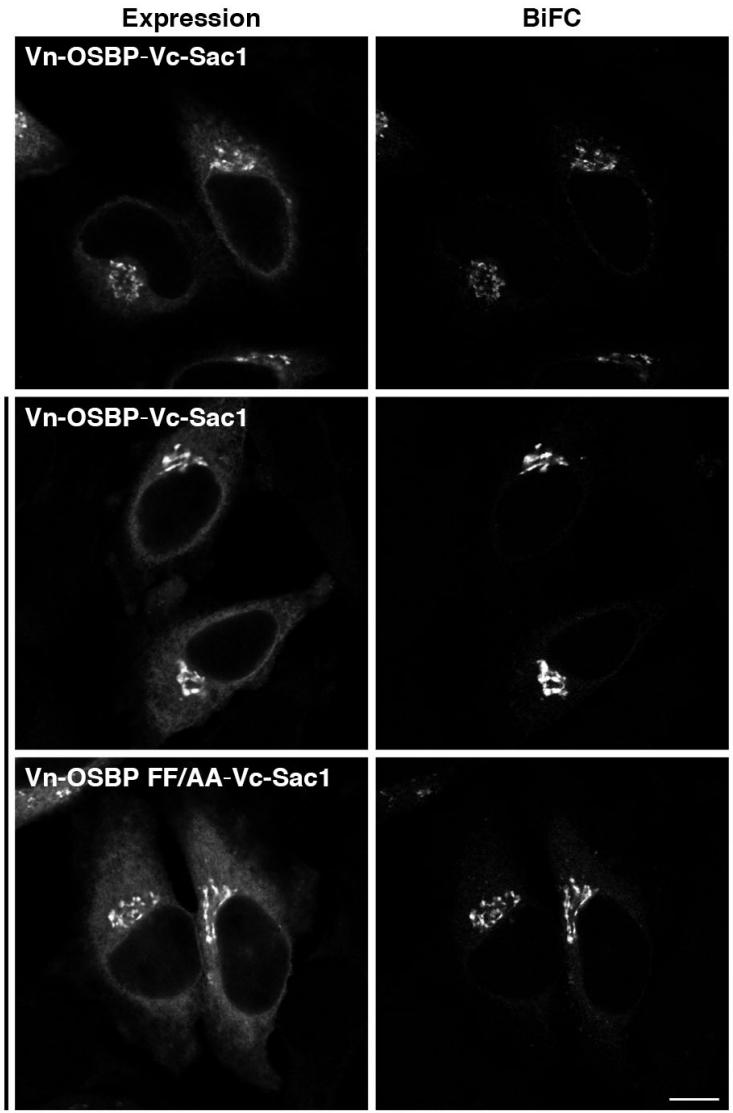

BiFC
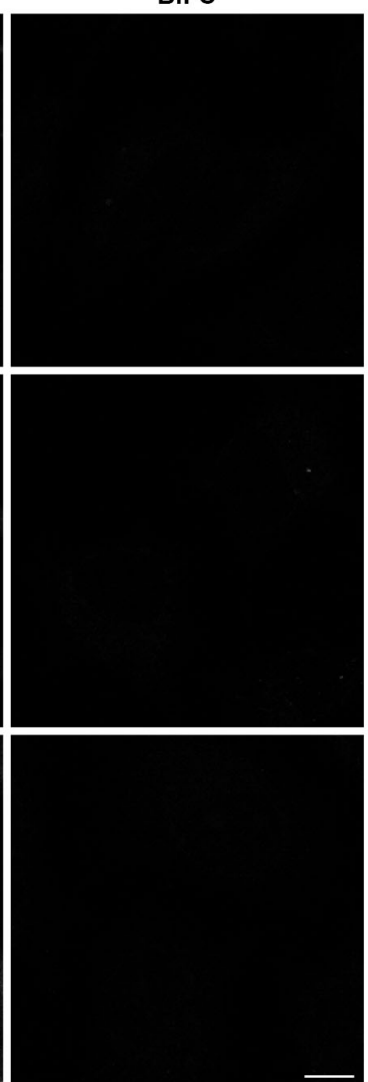

BiFC 
bioRxiv preprint doi: https://doi.org/10.1101/679936; this version posted February 28, 2020. The copyright holder for this preprint (which

was not certified by peer review) is the author/funder, who has granted bioRxiv a license to display the preprint in perpetuity. It is made available under aCC-BY-NC-ND 4.0 International license.

\section{Supplementary Fig. 3}

a

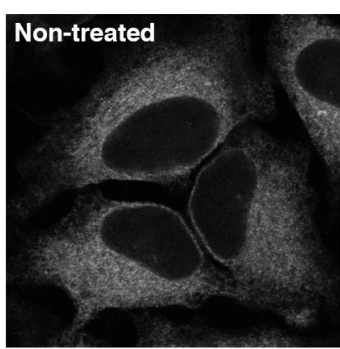

b
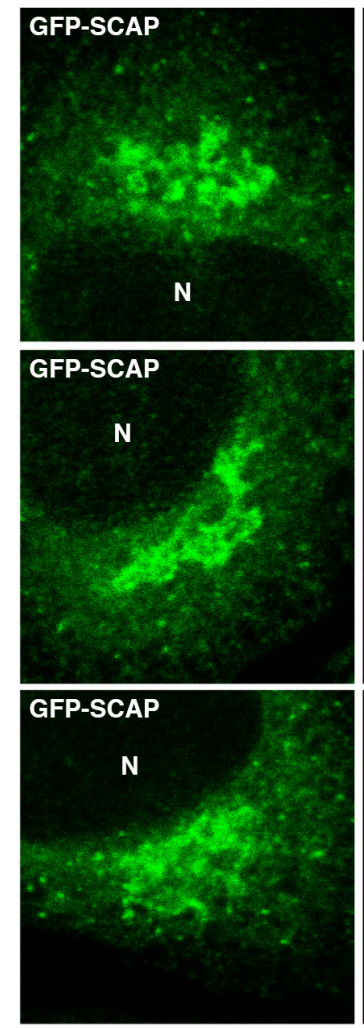

C

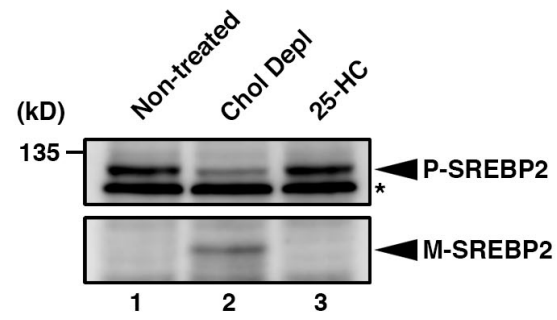

d
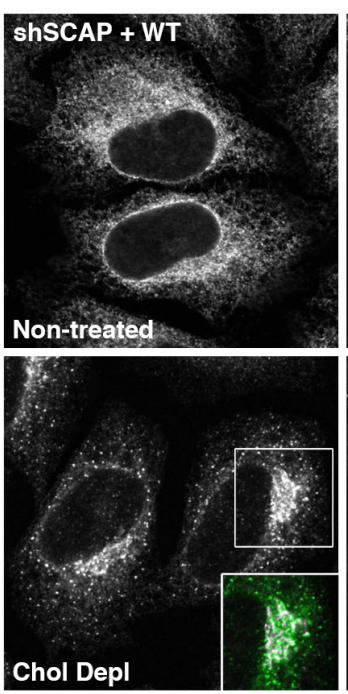
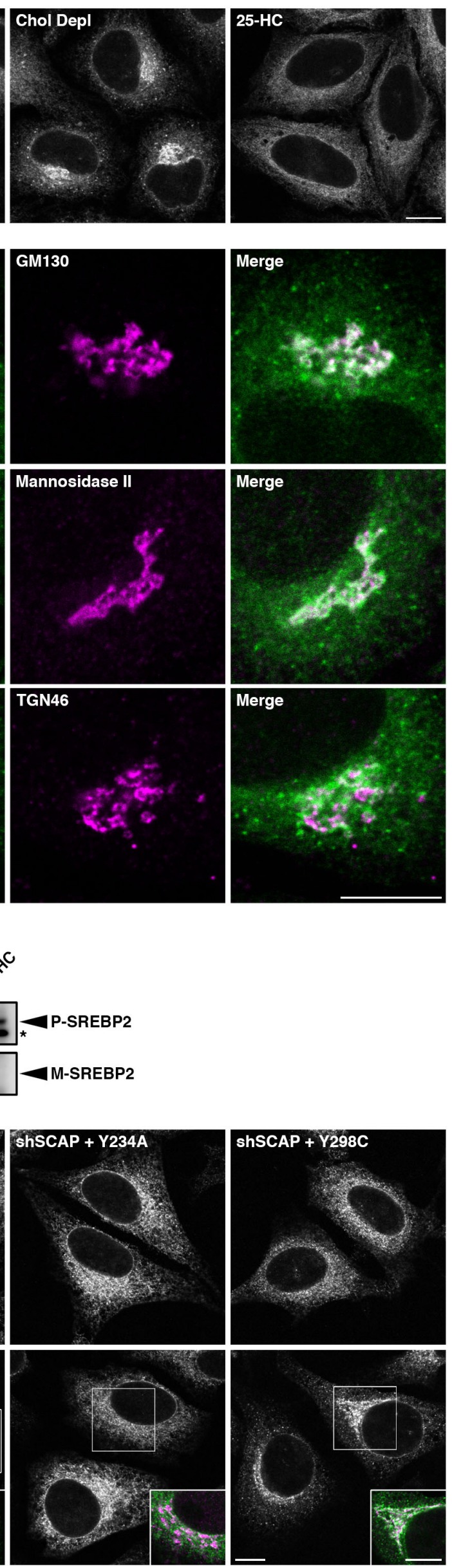
bioRxiv preprint doi: https://doi.org/10.1101/679936; this version posted February 28, 2020. The copyright holder for this preprint (which was not certified by peer review) is the author/funder, who has granted bioRxiv a license to display the preprint in perpetuity. It is made available under aCC-BY-NC-ND 4.0 International license.

\section{Supplementary Fig. 4}

a

PI4P
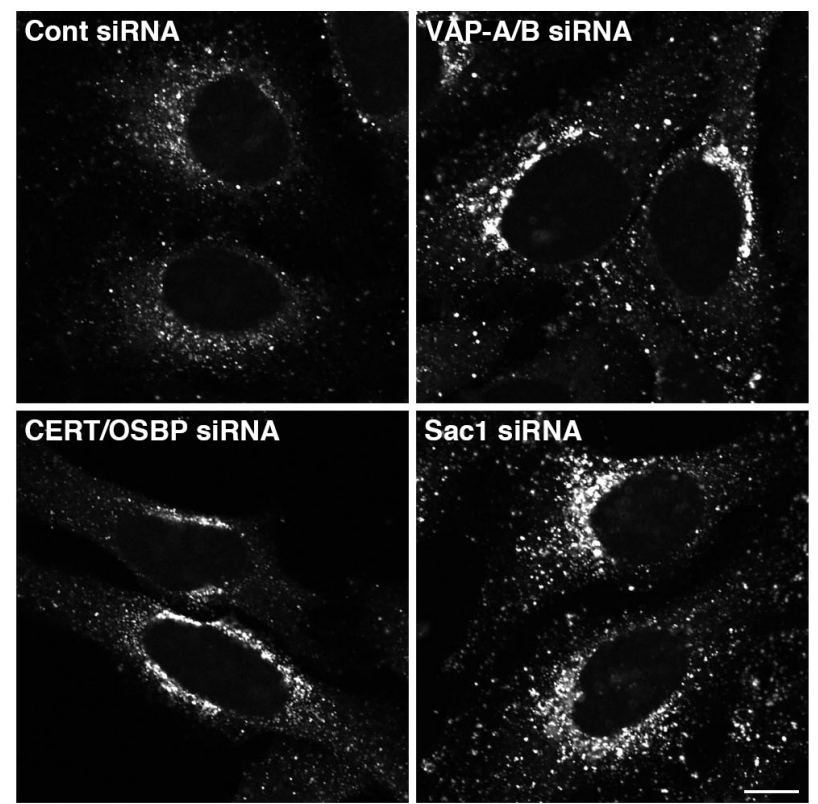

b

\section{Vn-OSBP-Vc-VAP-A}

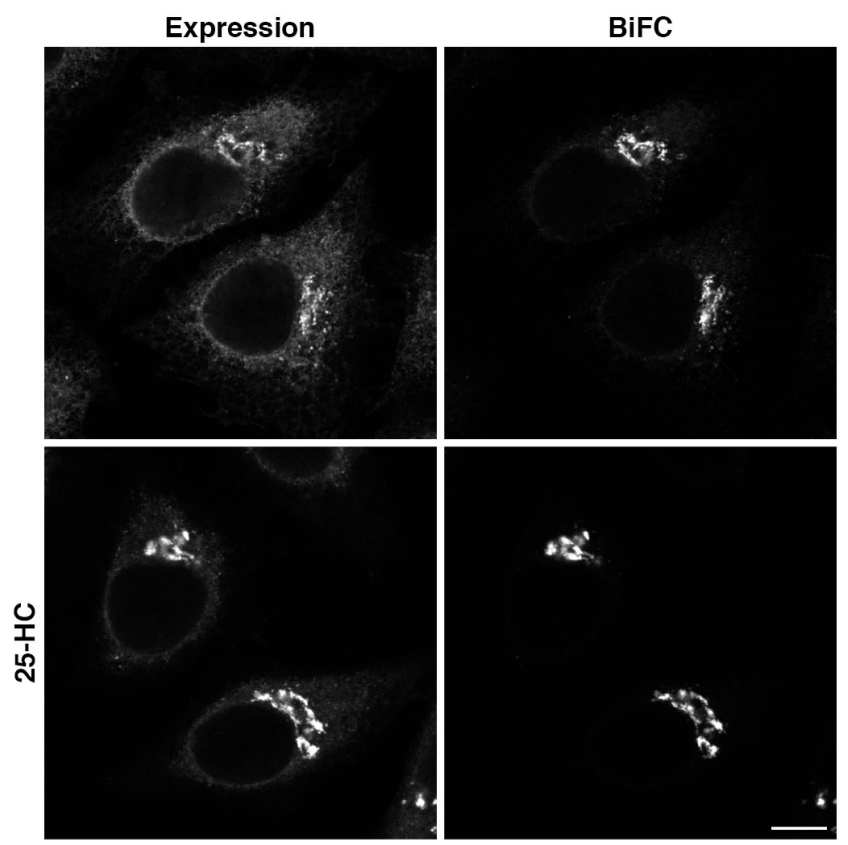


bioRxiv preprint doi: https://doi.org/10.1101/679936; this version posted February 28, 2020. The copyright holder for this preprint (which

was not certified by peer review) is the author/funder, who has granted bioRxiv a license to display the preprint in perpetuity. It is made available under aCC-BY-NC-ND 4.0 International license.

\section{Supplementary Fig. 5}

a
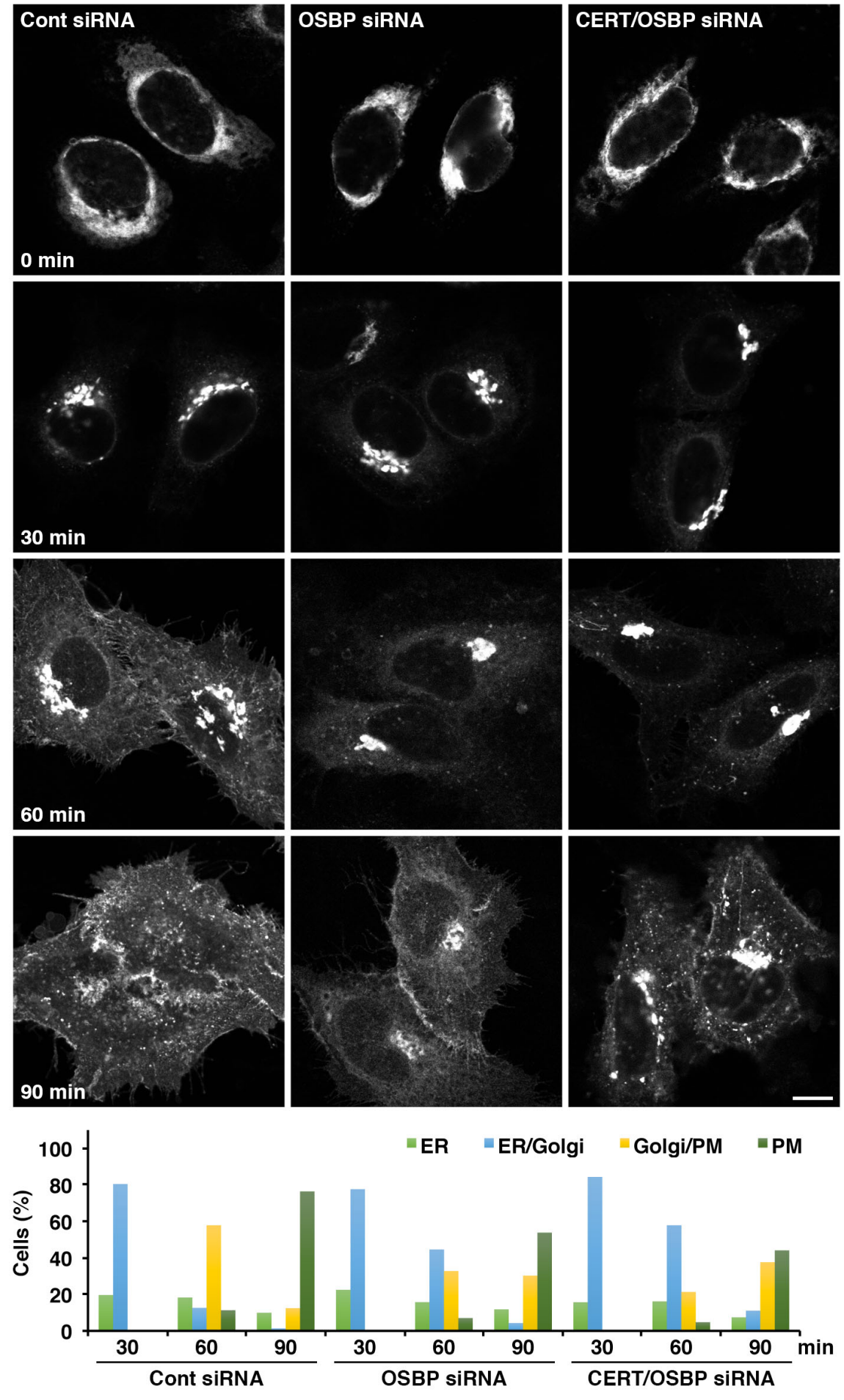

b

Myc-OSBP PH-FFAT
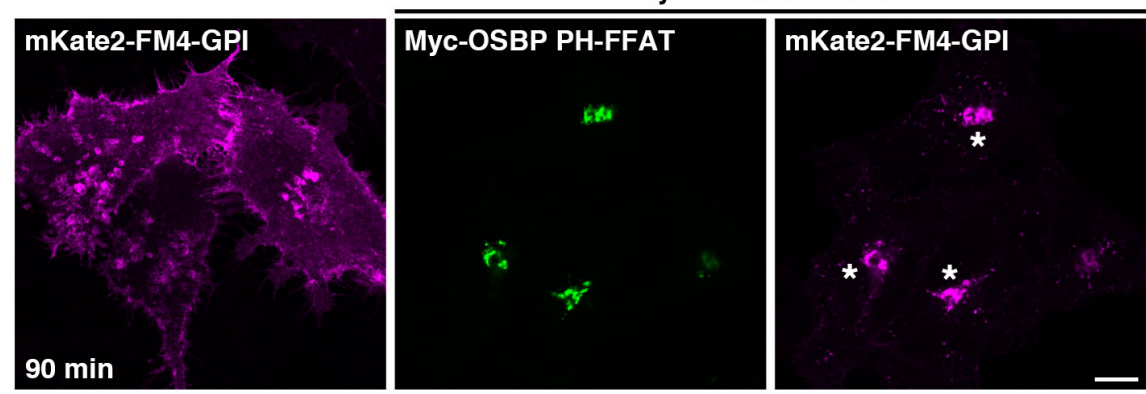